\title{
Commutative Algebra in the Cohomology of Groups
}

\author{
DAVE BENSON
}

\begin{abstract}
Commutative algebra is used extensively in the cohomology of groups. In this series of lectures, I concentrate on finite groups, but I also discuss the cohomology of finite group schemes, compact Lie groups, $p$-compact groups, infinite discrete groups and profinite groups. I describe the role of various concepts from commutative algebra, including finite generation, Krull dimension, depth, associated primes, the Cohen-Macaulay and Gorenstein conditions, local cohomology, Grothendieck's local duality, and Castelnuovo-Mumford regularity.
\end{abstract}

\section{Contents}

1. Introduction 2

2. Some Examples 2

3. Group Cohomology 8

4. Finite Generation $\quad 12$

5. Krull Dimension $\quad 15$

6. Depth 16

7. Associated Primes and Steenrod Operations 19

8. Associated Primes and Transfer 22

9. Idempotent Modules and Varieties 23

10. Modules with Injective Cohomology 26

11. Duality Theorems $\quad 30$

12. More Duality Theorems 34

13. Dual Localization 36

14. Quasiregular Sequences 38

Appendix: Two-Groups of Order 64 and Their mod 2 Cohomology 43

Acknowledgements $\quad 45$

$\begin{array}{ll}\text { References } & 45\end{array}$

The author is partly supported by NSF grant DMS-9988110. 


\section{Introduction}

The purpose of these lectures is to explain how commutative algebra is used in the cohomology of groups. My interpretation of the word "group" is catholic: the kinds of groups in which I shall be interested include finite groups, finite group schemes, compact Lie groups, $p$-compact groups, infinite discrete groups, and profinite groups, although later in the lectures I shall concentrate more on the case of finite groups, where representation theoretic methods are most effective. In each case, there are finite generation theorems which state that under suitable conditions, the cohomology ring is a graded commutative Noetherian ring; over a field $k$, this means that it is a finitely generated graded commutative $k$-algebra.

Although graded commutative is not quite the same as commutative, the usual concepts from commutative algebra apply. These include the maximal/prime ideal spectrum, Krull dimension, depth, associated primes, the Cohen-Macaulay and Gorenstein conditions, local cohomology, Grothendieck's local duality, and so on. One of the themes of these lectures is that the rings appearing in group cohomology theory are quite special. Most finitely generated graded commutative $k$-algebras are not candidates for the cohomology ring of a finite (or compact Lie, or virtual duality, or $p$-adic Lie, or ...) group. The most powerful restrictions come from local cohomology spectral sequences such as the Greenlees spectral sequence $H_{\mathfrak{m}}^{s, t} H^{*}(G, k) \Longrightarrow H_{-s-t}(G, k)$, which can be viewed as a sort of duality theorem. We describe how to construct such spectral sequences and obtain information from them.

The companion article to this one, [Iyengar 2004], explains some of the background material that may not be familiar to commutative algebraists. A number of references are made to that article, and for distinctiveness, I write [Sri].

\section{Some Examples}

For motivation, let us begin with some examples. We defer until the next section the definition of group cohomology

$$
H^{*}(G, k)=\operatorname{Ext}_{k G}^{*}(k, k)
$$

(or see $\S 6$ of [Sri]). All the examples in this section are for finite groups $G$ over a field of coefficients $k$.

(2.1) The first comment is that in the case where $k$ is a field of characteristic zero or characteristic not dividing the order of $G$, Maschke's theorem in representation theory shows that all $k G$-modules are projective (see Theorem 3.1 of [Sri]). So for any $k G$-modules $M$ and $N$, and all $i>0$, we have $\operatorname{Ext}_{k G}^{i}(M, N)=0$. In particular, $H^{*}(G, k)$ is just $k$, situated in degree zero. Given this fact, it makes sense to look at examples where $k$ has characteristic $p$ dividing $|G|$. 
(2.2) Next, we discuss finite abelian groups. See also $\S 7.4$ of [Sri]. The Künneth theorem implies that

$$
H^{*}\left(G_{1} \times G_{2}, k\right) \cong H^{*}\left(G_{1}, k\right) \otimes_{k} H^{*}\left(G_{2}, k\right) .
$$

So we decompose our finite abelian group as a direct product of cyclic groups of prime power order. The factors of order coprime to the characteristic may be thrown away, using (2.1). For a cyclic $p$-group in characteristic $p$, there are two possibilities (Proposition 7.3 of [Sri]). If $p=2$ and $|G|=2$, then $H^{*}(G, k)=k[x]$ where $x$ has degree one. In all other cases (i.e., $p$ odd, or $p=2$ and $|G| \geq 4$ ), we have $H^{*}(G, k)=k[x, y] /\left(x^{2}\right)$ where $x$ has degree one and $y$ has degree two. It follows that if $G$ is any finite abelian group then $H^{*}(G, k)$ is a tensor product of a polynomial ring and a (possibly trivial) exterior algebra.

(2.2.2) In particular, if $G$ is a finite elementary abelian $p$-group of rank $r$ (i.e., a product of $r$ copies of $\mathbb{Z} / p$ ) and $k$ is a field of characteristic $p$, then the cohomology ring is as follows. For $p=2$, we have

$$
H^{*}\left((\mathbb{Z} / 2)^{r}, k\right)=k\left[x_{1}, \ldots, x_{r}\right]
$$

with $\left|x_{i}\right|=1$, while for $p$ odd, we have

$$
H^{*}\left((\mathbb{Z} / p)^{r}, k\right)=\Lambda\left(x_{1}, \ldots, x_{r}\right) \otimes k\left[y_{1}, \ldots, y_{r}\right]
$$

with $\left|x_{i}\right|=1$ and $\left|y_{i}\right|=2$. In the latter case, the nil radical is generated by $x_{1}, \ldots, x_{r}$, and in both cases the quotient by the nil radical is a polynomial ring in $r$ generators.

(2.3) The next comment is that if $S$ is a Sylow $p$-subgroup of $G$ then a transfer argument shows that the restriction map from $H^{*}(G, k)$ to $H^{*}(S, k)$ is injective. What's more, the stable element method of Cartan and Eilenberg [1956] identifies the image of this restriction map. For example, if $S \unlhd G$ then $H^{*}(G, k)=$ $H^{*}(S, k)^{G / S}$, the invariants of $G / S$ acting on the cohomology of $S$ (see $\S 7.6$ of [Sri]). It follows that really important case is where $G$ is a $p$-group and $k$ has characteristic $p$. Abelian $p$-groups are discussed in (2.2), so let's look at some nonabelian $p$-groups.

Consider the quaternion group of order eight,

There is an embedding

$$
Q_{8}=\left\langle g, h \mid g h=h^{-1} g=h g^{-1}\right\rangle .
$$

$$
g \mapsto i, \quad h \mapsto j, \quad g h \mapsto k, \quad g^{2}=h^{2}=(g h)^{2} \mapsto-1
$$

of $Q_{8}$ into the unit quaternions (i.e., $\mathrm{SU}(2)$ ), which form a three dimensional sphere $S^{3}$. So left multiplication gives a free action of $Q_{8}$ on $S^{3}$; in other words, each nonidentity element of the group has no fixed points on the sphere. The quotient $S^{3} / Q_{8}$ is an orientable three dimensional manifold, whose cohomology 
therefore satisfies Poincaré duality. The freeness of the action implies that we can choose a CW decomposition of $S^{3}$ into cells permuted freely by $Q_{8}$. Taking cellular chains with coefficients in $\mathbb{F}_{2}$, we obtain a complex of free $\mathbb{F}_{2} Q_{8}$-modules of length four, whose homology consists of one copy of $\mathbb{F}_{2}$ at the beginning and another copy at the end. Making suitable choices for the cells, this looks as follows.

$$
0 \rightarrow \mathbb{F}_{2} Q_{8} \stackrel{\left(\begin{array}{l}
g-1 \\
h-1
\end{array}\right)}{\longrightarrow}\left(\mathbb{F}_{2} Q_{8}\right)^{2} \stackrel{\left(\begin{array}{c}
h-1 \\
g h+1
\end{array} \stackrel{h g+1}{g-1}\right)}{\longrightarrow}\left(\mathbb{F}_{2} Q_{8}\right)^{2} \stackrel{(g-1 h-1)}{\longrightarrow} \mathbb{F}_{2} Q_{8} \rightarrow 0
$$

So we can form a Yoneda splice of an infinite number of copies of this sequence to obtain a free resolution of $\mathbb{F}_{2}$ as an $\mathbb{F}_{2} Q_{8}$-module. The upshot of this is that we obtain a decomposition for the cohomology ring

$$
\begin{aligned}
H^{*}\left(Q_{8}, \mathbb{F}_{2}\right) & =\mathbb{F}_{2}[z] \otimes_{\mathbb{F}_{2}} H^{*}\left(S^{3} / Q_{8} ; \mathbb{F}_{2}\right) \\
& =\mathbb{F}_{2}[x, y, z] /\left(x^{2}+x y+y^{2}, x^{2} y+x y^{2}\right),
\end{aligned}
$$

where $z$ is a polynomial generator of degree four and $x$ and $y$ have degree one. This structure is reflected in the Poincaré series

$$
\sum_{i=0}^{\infty} t^{i} \operatorname{dim} H^{i}\left(Q_{8}, \mathbb{F}_{2}\right)=\left(1+2 t+2 t^{2}+t^{3}\right) /\left(1-t^{4}\right)
$$

The decomposition (2.4.2) into a polynomial piece and a finite Poincaré duality piece can be expressed as follows (cf. $\S 11)$ :

$$
H^{*}\left(Q_{8}, \mathbb{F}_{2}\right) \text { IS A Gorenstein RING. }
$$

(2.5) We recall that the meanings of Cohen-Macaulay and Gorenstein in this context are as follows. Let $R$ be a finitely generated graded commutative $k$ algebra with $R_{0}=k$ and $R_{i}=0$ for $i<0$. Then Noether's normalization lemma guarantees the existence of a homogeneous polynomial subring $k\left[x_{1}, \ldots, x_{r}\right]$ over which $R$ is finitely generated as a module.

Proposition 2.5.1. If $R$ is of the type described in the previous paragraph, then the following are equivalent.

(a) There exists a homogeneous polynomial subring $k\left[x_{1}, \ldots, x_{r}\right] \subseteq R$ such that $R$ is finitely generated and free as a module over $k\left[x_{1}, \ldots, x_{r}\right]$.

(b) If $k\left[x_{1}, \ldots, x_{r}\right] \subseteq R$ is a homogeneous polynomial subring such that $R$ is finitely generated as a $k\left[x_{1}, \ldots, x_{r}\right]$-module then $R$ a free $k\left[x_{1}, \ldots, x_{r}\right]$-module.

(c) There exist homogeneous elements of positive degree $x_{1}, \ldots, x_{r}$ forming a regular sequence, and $R /\left(x_{1}, \ldots, x_{r}\right)$ has finite rank as a $k$-vector space.

We say that $R$ is Cohen-Macaulay of dimension $r$ if the equivalent conditions of the above proposition hold. 
(2.6) If $R$ is Cohen-Macaulay, and the quotient ring $R /\left(x_{1}, \ldots, x_{r}\right)$ has a simple socle, then we say that $R$ is Gorenstein. Whether this condition holds is independent of the choice of the polynomial subring. Another way to phrase the condition is that $R /\left(x_{1}, \ldots, x_{r}\right)$ is injective as a module over itself. This quotient satisfies Poincaré duality, in the sense that if the socle lies in degree $d$ ( $d$ is called the dualizing degree) and we write

$$
p(t)=\sum_{i=0}^{\infty} t^{i} \operatorname{dim}_{k}\left(R /\left(x_{1}, \ldots, x_{r}\right)\right)_{i}
$$

then

$$
t^{d} p(1 / t)=p(t)
$$

Setting

$$
P(t)=\sum_{i=0}^{\infty} t^{i} \operatorname{dim}_{k} R_{i}
$$

the freeness of $R$ over $k\left[x_{1}, \ldots, x_{r}\right]$ implies that $P(t)$ is the power series expansion of the rational function $p(t) / \prod_{i=1}^{r}\left(1-t^{\left|x_{i}\right|}\right)$. So plugging in equation (2.6.1), we obtain the functional equation

$$
P(1 / t)=(-t)^{r} t^{-a} P(t),
$$

where $a=d-\sum_{i=1}^{r}\left(\left|x_{i}\right|-1\right)$. We say that $R$ is Gorenstein with $a$-invariant $a$.

Another way of expressing the Gorenstein condition is as follows. If $R$ (as above) is Cohen-Macaulay, then the local cohomology $H_{\mathfrak{m}}^{s, t} R$ is only nonzero for $s=r$. The graded dual of $H_{\mathfrak{m}}^{r, *} R$ is called the canonical module, and written $\Omega_{R}$. To say that $R$ is Gorenstein with $a$-invariant $a$ is the same as saying that $\Omega_{R}$ is a copy of $R$ shifted so that the identity element lies in degree $r-a$.

In the case of $H^{*}\left(Q_{8}, \mathbb{F}_{2}\right)$, we can choose the polynomial subring to be $k[z]$. The ring $H^{*}\left(Q_{8}, \mathbb{F}_{2}\right)$ is a free module over $k[z]$ on six generators, corresponding to a basis for the graded vector space $H^{*}\left(S^{3} / Q_{8} ; \mathbb{F}_{2}\right) \cong H^{*}\left(Q_{8}, \mathbb{F}_{2}\right) /(z)$, which satisfies Poincaré duality with $d=3$. So in this case the $a$-invariant is $3-(4-1)=$ 0 . We have $p(t)=1+2 t+2 t^{2}+t^{3}$ and $P(t)=p(t) /\left(1-t^{4}\right)$.

(2.7) A similar pattern to the one seen above for $Q_{8}$ holds for other groups. Take for example the group $\operatorname{GL}(3,2)$ of $3 \times 3$ invertible matrices over $\mathbb{F}_{2}$. This is a finite simple group of order 168. Its cohomology is given by

$$
H^{*}\left(\mathrm{GL}(3,2), \mathbb{F}_{2}\right)=\mathbb{F}_{2}[x, y, z] /\left(x^{3}+y z\right)
$$

where $\operatorname{deg} x=2, \operatorname{deg} y=\operatorname{deg} z=3$. A homogeneous system of parameters for this ring is given by $y$ and $z$, and these elements form a regular sequence. Modulo the ideal generated by $y$ and $z$, we get $\mathbb{F}_{2}(x) /\left(x^{3}\right)$. This is a finite Poincaré duality ring whose dualizing degree is 4 . Again, this means that the cohomology is a Gorenstein ring with $a$-invariant $4-(3-1)-(3-1)=0$, but it 
does not decompose as a tensor product the way it did for the quaternion group (2.4.2).

(2.8) It is not true that the cohomology ring of a finite group is always Gorenstein. For example, the semidihedral group of order $2^{n}(n \geq 4)$,

$$
G=S D_{2^{n}}=\left\langle g, h \mid g^{2^{n-1}}=1, h^{2}=1, h^{-1} g h=g^{2^{n-2}-1}\right\rangle
$$

has cohomology ring

$$
H^{*}\left(S D_{2^{n}}, \mathbb{F}_{2}\right)=\mathbb{F}_{2}[x, y, z, w] /\left(x y, y^{3}, y z, z^{2}+x^{2} w\right)
$$

with $\operatorname{deg} x=\operatorname{deg} y=1, \operatorname{deg} z=3$ and $\operatorname{deg} w=4$. This ring is not even CohenMacaulay. But what is true is that whenever the ring is Cohen-Macaulay, it is Gorenstein with $a$-invariant zero. See $\S 11$ for further details.

Even if the cohomology ring is not Cohen-Macaulay, there is still a certain kind of duality, but it is expressed in terms of a spectral sequence of Greenlees, $H_{\mathfrak{m}}^{s, t} H^{*}(G, k) \Longrightarrow H_{-s-t}(G, k)$. Let us see in the case above of the semidihedral group, what this spectral sequence looks like. And let's do it in pictures. We'll draw the cohomology ring as follows.

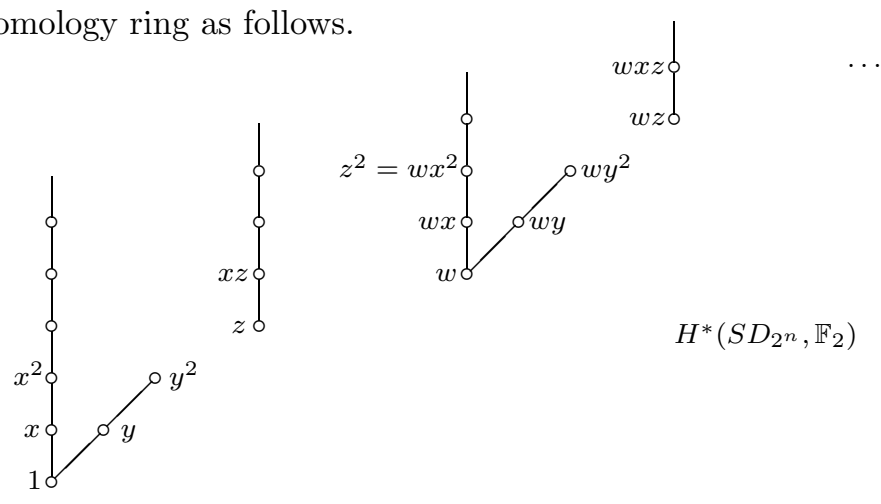

The vertical coordinate indicates cohomological degree, and the horizontal coordinate is just for separating elements of the same degree. To visualize the homology, just turn this picture upside down by rotating the page, as follows.

$$
H_{*}\left(S D_{2^{n}}, \mathbb{F}_{2}\right)
$$
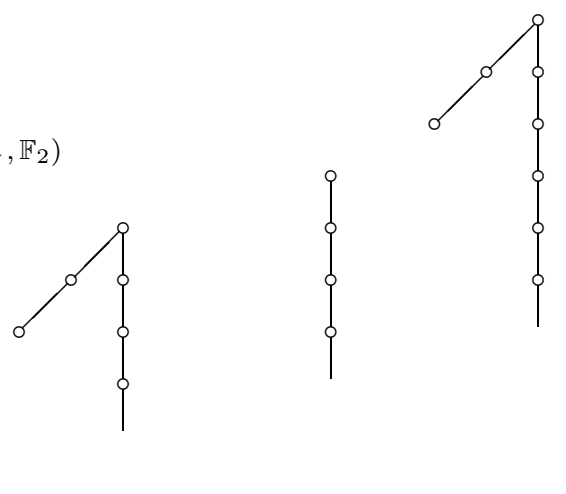
We compute local cohomology using the stable Koszul complex for the homogeneous system of parameters $w, x$,

$0 \rightarrow H^{*}\left(G, \mathbb{F}_{2}\right) \rightarrow H^{*}\left(G, \mathbb{F}_{2}\right)\left[w^{-1}\right] \oplus H^{*}\left(G, \mathbb{F}_{2}\right)\left[x^{-1}\right] \rightarrow H^{*}\left(G, \mathbb{F}_{2}\right)\left[w^{-1} x^{-1}\right] \rightarrow 0$

where the subscripts denote localization by inverting the named element. A picture of this stable Koszul complex is as follows.

0
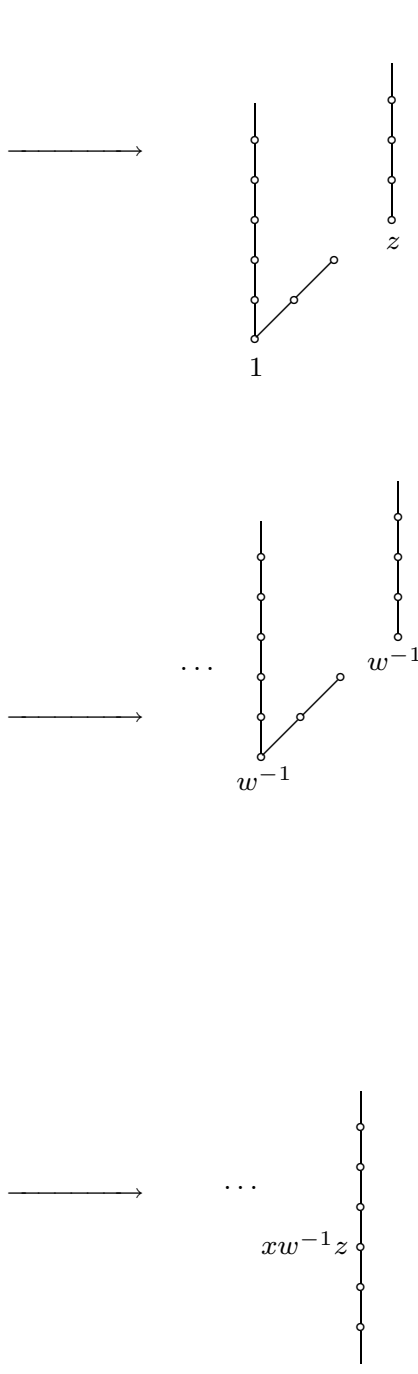
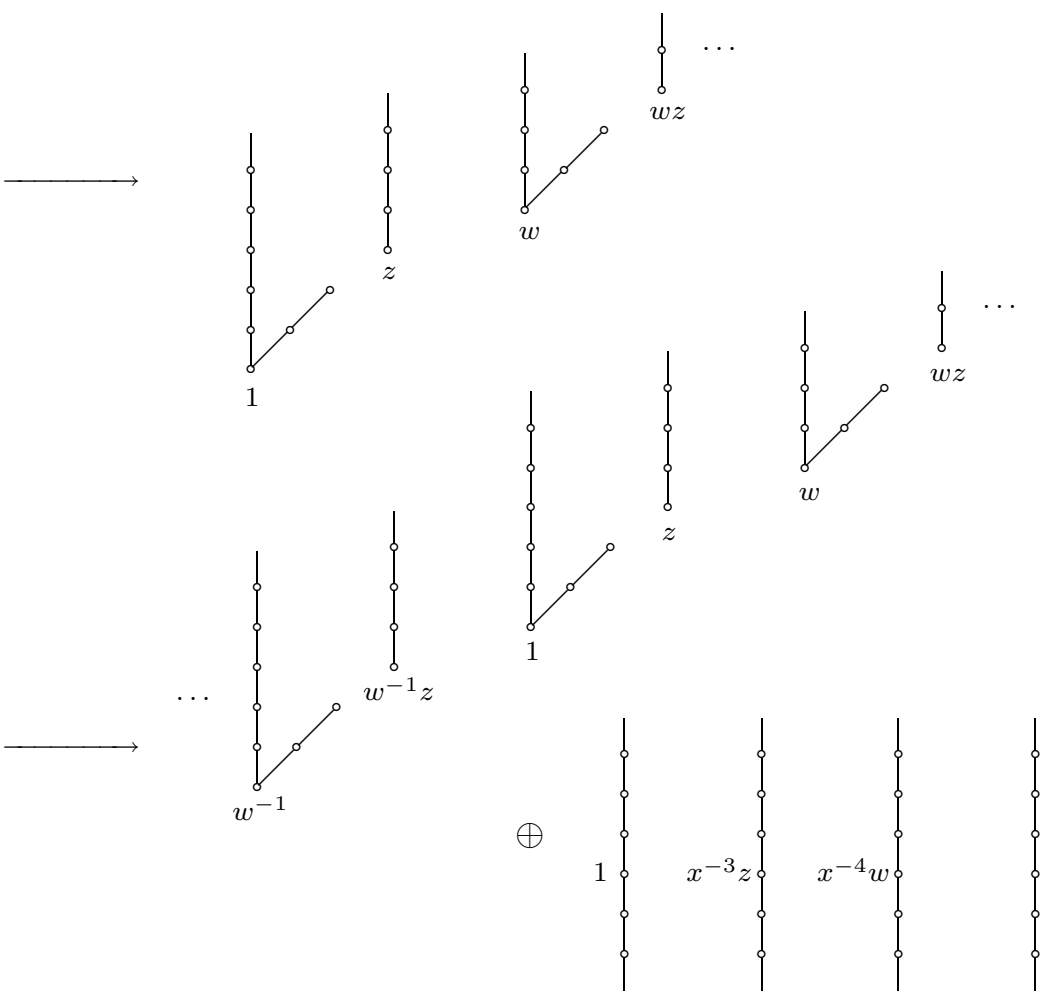

$\cdots \quad \longrightarrow 0$.

The local cohomology of $H^{*}(G, k)$ is just the cohomology of this complex. In degree zero there is no cohomology. In degree one there is some cohomology, namely the hooks that got introduced when $w$ was inverted, 


$$
\underset{w^{-1} y}{\int} w^{-1} y^{2}
$$

$$
H_{\mathfrak{m}}^{1} H^{*}\left(S D_{2^{n}}, \mathbb{F}_{2}\right)=\quad \ldots \quad \int_{w^{-2} y} \rho w^{-2} y^{2}
$$

In degree two, we get the part of the plane not hit by either of the two degree one pieces,

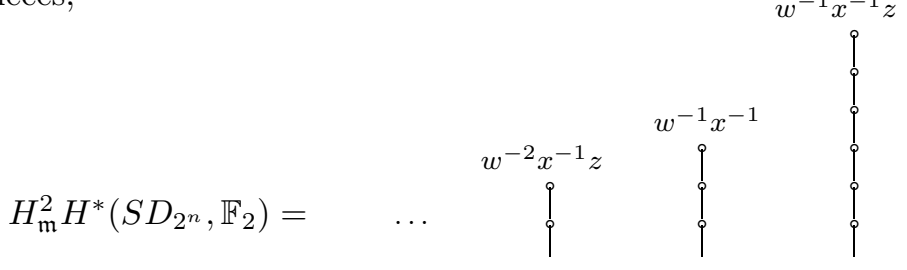

Now the differential $d_{2}$ in this spectral sequence increases local cohomological degree by two and decreases internal degree by one, and the higher differentials are only longer. So there is no room in this example for nonzero differentials. It follows that the spectral sequence takes the form of a short exact sequence

$$
0 \rightarrow H_{\mathfrak{m}}^{1, t-1} H^{*}\left(S D_{2^{n}}, \mathbb{F}_{2}\right) \rightarrow H_{-t}\left(S D_{2^{n}}, \mathbb{F}_{2}\right) \rightarrow H_{\mathfrak{m}}^{2, t-2} H^{*}\left(S D_{2^{n}}, \mathbb{F}_{2}\right) \rightarrow 0 .
$$

This works fine, because $H_{*}\left(S D_{2^{n}}, \mathbb{F}_{2}\right)$ is the graded dual of $H^{*}\left(S D_{2^{n}}, \mathbb{F}_{2}\right)$, as shown in (2.8.2). So the short exact sequence places the hooks of $H_{\mathfrak{m}}^{1}$ underneath every second nonzero column in $H_{\mathfrak{m}}^{2}$ to build $H_{*}\left(S D_{2^{n}}, \mathbb{F}_{2}\right)$. Notice that the hooks appear inverted, so that there is a separate Poincaré duality for a hook.

The same happens as in this case whenever the depth and the Krull dimension differ by one. The kernel of multiplication by the last parameter, modulo the previous parameters, satisfies Poincaré duality with dualizing degree determined by the degrees of the parameters; in particular, the top degree of this kernel is determined. In the language of commutative algebra, this can be viewed in terms of the Castelnuovo-Mumford regularity of the cohomology ring. See $\S 14$ for more details.

The reader who wishes to understand these examples better can skip directly to $\S 14$, and refer back to previous sections as necessary to catch up on definitions. Conjecture 14.6.1 says that for a finite group $G$, $\operatorname{Reg} H^{*}(G, k)$ is always zero. This conjecture is true when the depth and the Krull dimension differ by at most one, as in the above example. It is even true when the difference is two, by a more subtle transfer argument sketched in $\S 14$ and described in detail in [Benson 2004].

\section{Group Cohomology}

For general background material on cohomology of groups, the textbooks I recommend are [Adem and Milgram 1994; Benson 1991b; Brown 1982; Cartan and Eilenberg 1956; Evens 1991]. The commutative algebra texts most relevant 
to these lectures are [Bruns and Herzog 1993; Eisenbud 1995; Grothendieck 1965; 1967; Matsumura 1989].

(3.1) For a discrete group, the easiest way to think of group cohomology is as the Ext ring (see $\S 5$ of [Sri]). If $G$ is a group and $k$ is a commutative ring of coefficients, we define group cohomology via

$$
H^{*}(G, k)=\operatorname{Ext}_{\mathbb{Z} G}^{*}(\mathbb{Z}, k) \cong \operatorname{Ext}_{k G}^{*}(k, k)
$$

Here, the group ring $k G$ consists of formal linear combinations $\sum \lambda_{i} g_{i}$ of elements of the group $G$ with coefficients in $k$. The cup product in cohomology comes from the fact that $k G$ is a Hopf algebra (see $\S 1.8$ of [Sri]), with comultiplication $\Delta(g)=g \otimes g$. Another part of the Hopf structure on $k G$ is the augmentation map $k G \rightarrow k, \sum \lambda_{i} g_{i} \mapsto \sum \lambda_{i}$, which is what allows us to regard $k$ as a $k G$-module.

Cup product and Yoneda product define the same multiplicative structure, and this makes cohomology into a graded commutative ring, in the sense that

$$
a b=(-1)^{|a||b|} b a,
$$

where $|a|$ denotes the degree of an element $a$ (see Prop. 5.5 of [Sri]). In contrast, the Ext ring of a commutative local ring is seldom graded commutative; this happens only for a restricted class of complete intersections. The group ring of an abelian group is an example of a complete intersection (see $\S 1.4$ of [Sri]).

More generally, if $M$ is a left $k G$-module then

$$
H^{*}(G, M)=\operatorname{Ext}_{\mathbb{Z} G}^{*}(\mathbb{Z}, M) \cong \operatorname{Ext}_{k G}^{*}(k, M)
$$

is a graded right $H^{*}(G, k)$-module.

It is a nuisance that most texts on commutative algebra are written for strictly commutative graded rings, where $a b=b a$ with no sign. I do not know of an instance where the signs make a theorem from commutative algebra fail. It is worth pointing out that if $a$ is an element of odd degree in a graded commutative ring then $2 a^{2}=0$. So $2 a$ is nilpotent, and it follows that modulo the nil radical the ring is strictly commutative. On the other hand, it is more than a nuisance that commutative algebraists often assume that their graded rings are generated by elements of degree one, because this is not at all true for cohomology rings. Nor, for that matter, is it true for rings of invariants.

(3.2) A homomorphism of groups $\rho: H \rightarrow G$ gives rise to a map the other way

$$
\rho^{*}: H^{*}(G, M) \rightarrow H^{*}(H, M)
$$

for any $k G$-module $M$. If $\rho: H \rightarrow G$ is an inclusion, this is called the restriction map, and denoted $\operatorname{res}_{G, H}$. If $G$ is a quotient group of $H$ and $\rho: H \rightarrow G$ is the quotient map, then it is called the inflation map, and denoted $\inf _{G, H}$. 
(3.3) For a topological group (this includes compact Lie groups as well as discrete groups), a theorem of Milnor [1956] says that the infinite join

$$
E G=G \star G \star \cdots
$$

is weakly contractible, $G$ acts freely on it, and the quotient $B G=E G / G$ together with the principal $G$-bundle $p: E G \rightarrow B G$ forms a classifying space for principal $G$-bundles over a paracompact base. A topologist refers to $H^{*}(B G ; k)$ as the classifying space cohomology of $G$. Again, it is a graded commutative ring. For example, for the compact unitary group $\mathrm{U}(n)$, the cohomology ring

$$
H^{*}(B \mathrm{U}(n) ; k) \cong k\left[c_{1}, \ldots, c_{n}\right]
$$

is a polynomial ring over $k$ on $n$ generators $c_{1}, \ldots, c_{n}$ with $\left|c_{i}\right|=2 i$, called the Chern classes. Similarly, for the orthogonal group $\mathrm{O}(2 n)$, if $k$ is a field of characteristic not equal to two, then we have

$$
H^{*}(B \mathrm{O}(2 n) ; k) \cong k\left[p_{1}, \ldots, p_{n}\right]
$$

is a polynomial ring over $k$ on $n$ generators $p_{1}, \ldots, p_{n}$ with $\left|p_{i}\right|=4 i$, called the Pontrjagin classes. For $\mathrm{SO}(2 n)$ we have

$$
H^{*}(B \operatorname{SO}(2 n) ; k) \cong k\left[p_{1}, \ldots, p_{n-1}, e\right] .
$$

where $e \in H^{2 n}(B \mathrm{SO}(2 n) ; k)$ is called the Euler class, and satisfies $e^{2}=p_{n}$. We shall discuss these examples further in $\S 12$.

If $G$ is a discrete group then $B G$ is an Eilenberg-Mac Lane space for $G$; in other words, $\pi_{1}(B G) \cong G$ and $\pi_{i}(B G)=0$ for $i>1$. The relationship between group cohomology and classifying space cohomology for $G$ discrete is that the singular chains $C_{*}(E G)$ form a free resolution of $\mathbb{Z}$ as a $\mathbb{Z} G$-module. Then there are isomorphisms

$$
H^{*}(B G ; k)=H^{*} \operatorname{Hom}_{\mathbb{Z}}\left(C_{*}(B G), k\right) \cong H^{*} \operatorname{Hom}_{\mathbb{Z} G}\left(C_{*}(E G), k\right) \cong H^{*}(G, k),
$$

and the topologically defined product on the left agrees with the algebraically defined product on the right.

(3.4) Another case of interest is profinite groups. A profinite group is defined to be an inverse limit of a system of finite groups, which makes it a compact, Hausdorff, totally disconnected topological group. For example, writing $\mathbb{Z}_{p}^{\wedge}$ for the ring of $p$-adic integers, $\mathrm{SL}_{n}\left(\mathbb{Z}_{p}^{\wedge}\right)$ is a profinite group. The open subgroups of a profinite group are the subgroups of finite index.

Classifying space cohomology turns out to be the wrong concept for a profinite group. A better concept is continuous cohomology, which is defined as follows [Serre 1965a]. Let $G=\lim _{U \in \mathscr{U}} G / U$ be a profinite group, where $\mathscr{U}$ is a system of 
open normal subgroups with $\bigcap_{U \in \mathscr{U}} U=\{1\}$. We restrict attention to modules $M$ such that $M=\bigcup_{U \in \mathscr{U}} M^{U}$, and continuous cohomology is then defined as

$$
H_{c}^{i}(G, M)=\lim _{U \in \mathscr{U}} H^{i}\left(G / U, M^{U}\right) .
$$

Again, if $k$ is a commutative ring of coefficients then $H_{c}^{*}(G, k)$ is a graded commutative ring.

(3.5) In all of the above situations, if $p$ is a prime number and $k$ is the finite field $\mathbb{F}_{p}$, then there are Steenrod operations

$$
\begin{cases}\mathrm{Sq}^{i}: H^{n}\left(G, \mathbb{F}_{2}\right) \rightarrow H^{n+i}\left(G, \mathbb{F}_{2}\right) & (p=2), \\ P^{i}: H^{n}\left(G, \mathbb{F}_{p}\right) \rightarrow H^{n+2 i(p-1)}\left(G, \mathbb{F}_{p}\right) & (p \text { odd })\end{cases}
$$

$(i \geq 0)$ acting on the cohomology of any group $\left(\mathrm{Sq}^{0}\right.$ and $P^{0}$ act as the identity operation). ${ }^{1}$ These operations satisfy some identities called the Adem relations, and the Steenrod algebra is the graded algebra generated by the Steenrod operations subject to the Adem relations. The action of the Steenrod operations is related to the multiplicative structure of cohomology by the Cartan formula

$$
\begin{cases}\mathrm{Sq}^{n}(x y)=\sum_{i+j=n} \mathrm{Sq}^{i}(x) \mathrm{Sq}^{j}(y) & (p=2), \\ P^{n}(x y)=\sum_{i+j=n} P^{i}(x) P^{j}(y) & (p \text { odd })\end{cases}
$$

Finally, the action of the Steenrod operations on group cohomology satisfies the unstable axiom, which states that

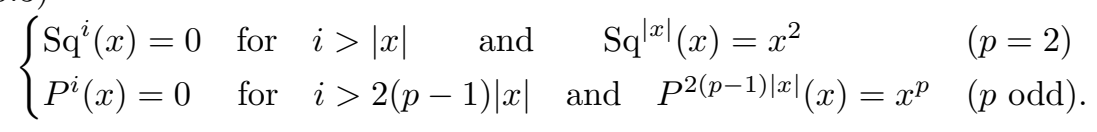

The Cartan formula and the unstable axiom make the cohomology ring of a group (or more generally, the cohomology ring of any space) with $\mathbb{F}_{p}$ coefficients into an unstable algebra over the Steenrod algebra. For more details, see [Steenrod 1962; Schwartz 1994].

(3.6) There are some important variations on the definitions of group cohomology. For example, for a finite group, Tate cohomology is defined using complete resolutions, and gives a $\mathbb{Z}$-graded ring $\hat{H}^{*}(G, k)$. More precisely, if $G$ is a finite group, $k$ is a field ${ }^{2}$ and $N$ is a $k G$-module, then we splice together an injective

\footnotetext{
${ }^{1}$ For $p$ odd, there is also a separate Bockstein operation $\beta: H^{n}\left(G, \mathbb{F}_{p}\right) \rightarrow H^{n+1}\left(G, \mathbb{F}_{p}\right)$ which we shall systematically ignore. For $p=2$, the Bockstein operation is equal to $\mathrm{Sq}^{1}$, so it is not a separate operation.

${ }^{2}$ Tate cohomology is defined over an arbitrary commutative ring of coefficients, but the definition is slightly different to the one given here. See [Cartan and Eilenberg 1956, Chapter $\mathrm{XII}]$.
} 
resolution and a projective resolution of $N$ to give an exact sequence

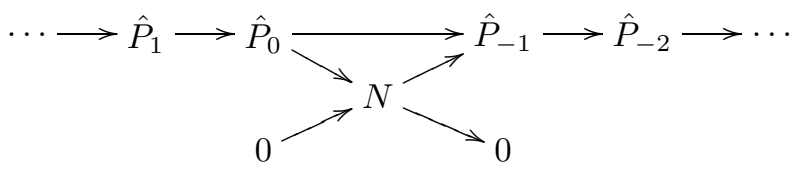

The fact that injective $k G$-modules are the same as projective $k G$-modules (see Theorem 3.6 as well as the paragraph following Corollary 3.7 in [Sri]) means that this is an exact sequence of projective modules such that the image of the middle map is equal to $N$, which is the definition of a complete resolution. If $M$ is another $k G$-module, we define $\widehat{\operatorname{Ext}}_{k G}^{*}(N, M)$ to be the cohomology of the cochain complex obtained by taking homomorphisms from the complete resolution $\hat{P}_{*}$ to $M$. In the case where $N=k$, we define

$$
\hat{H}^{*}(G, M)=\widehat{\operatorname{Ext}}_{k G}^{*}(k, M) .
$$

If $M$ is also equal to $k$, then $\hat{H}^{*}(G, k)$ is a graded commutative ring. If $M$ is a left $k G$-module then $\hat{H}^{*}(G, M)$ is a right module over $\hat{H}^{*}(G, k)$.

There is a map from a complete resolution of $N$ to the projective resolution of $N$ used to make it

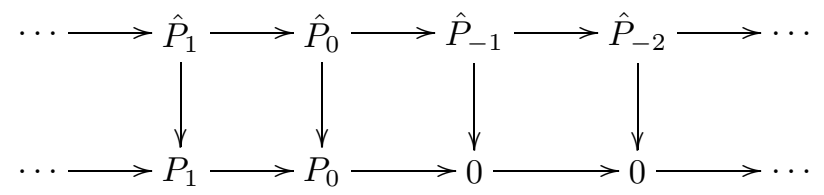

which is an isomorphism in nonnegative degrees and the zero map in negative degrees. This induces a map from $\operatorname{Ext}_{k G}^{*}(N, M)$ to $\widehat{\operatorname{Ext}}_{k G}^{*}(N, M)$ which is an isomorphism in positive degrees, surjective in degree zero, and the zero map in negative degrees. In particular, we obtain this way a map from ordinary cohomology to Tate cohomology, $H^{*}(G, M) \rightarrow \hat{H}^{*}(G, M)$. This is a homomorphism of graded $k$-algebras.

Tate duality says that for any $k G$-module $M$ and every $n \in \mathbb{Z}$, the $k$-vector space $\widehat{\operatorname{Ext}}_{k G}^{n-1}(M, k)$ is the dual of $\hat{H}^{-n}(G, M)$,

$$
\operatorname{Ext}_{k G}^{n-1}(M, k) \cong \operatorname{Hom}_{k}\left(\hat{H}^{-n}(G, M), k\right)
$$

The case $M=k$ of this statement can be interpreted as saying that the Tate cohomology is isomorphic to its graded dual, shifted in degree by one. This implies that it is selfinjective as a ring.

\section{Finite Generation}

There are various finite generation theorems, which provide the Noetherian hypothesis as starting point for the application of commutative algebra. 
(4.1) We begin with finite groups, where we have the following algebraic theorem of Evens [1961] (see also [Golod 1959] for the case of a finite $p$-group).

THEOREM 4.1.1. Let $G$ be a finite group, $k$ a commutative ring of coefficients and $M$ a $k G$-module. If $M$ is Noetherian as a $k$-module then $H^{*}(G, M)$ is Noetherian as an $H^{*}(G, k)$-module. In particular, if $k$ is Noetherian then $H^{*}(G, k)$ is a finitely generated $k$-algebra.

This can be contrasted with the situation in commutative algebra, where the Ext ring of a commutative local ring is Noetherian if and only if the ring is a complete intersection [Bøgvad and Halperin 1986]. The following extension of the theorem above also appears in [Evens 1961].

THEOREM 4.1.2. Let $H$ be a subgroup of a finite group $G$, let $k$ be a commutative ring of coefficients, and let $N$ be a Noetherian $k H$-module. Then $H^{*}(H, N)$ is a finitely generated module over $H^{*}(G, k)$ via the restriction map (3.2) from $G$ to $H$.

In contrast, Tate cohomology is almost never finitely generated. In fact, if $k$ is a field of characteristic $p$, then there is a dichotomy [Benson and Krause 2002]. Either

(4.1.3) $G$ has no subgroups isomorphic to $\mathbb{Z} / p \times \mathbb{Z} / p$ (i.e., the Sylow $p$-subgroups of $G$ are cyclic, or $p=2$ and the Sylow 2-subgroups of $G$ are generalized quaternion) and $\hat{H}^{*}(G, k)$ is periodic and Noetherian, of the form $k\left[x, x^{-1}\right]$ tensored with a finite dimensional part, or

(4.1.4) $G$ has a subgroup isomorphic to $\mathbb{Z} / p \times \mathbb{Z} / p$ and $\hat{H}^{*}(G, k)$ is not Noetherian. In this case, the negative degree cohomology $\hat{H}^{-}(G, k)$ is nilpotent, in the sense that there is some integer $n$ such that every product of $n$ or more elements of $\hat{H}^{-}(G, k)$ gives zero. In fact, if the depth of $H^{*}(G, k)$ is bigger than one then all products in $\hat{H}^{-}(G, k)$ vanish [Benson and Carlson 1992].

(4.2) Evens' theorem generalizes in a number of directions. The following is a theorem of Friedlander and Suslin [1997].

TheOrem 4.2.1. Let $G$ be a finite group scheme over a field $k$ (i.e., $k G$ is a finite dimensional cocommutative Hopf algebra), and let $M$ be a finitely generated $k G$-module. Then $H^{*}(G, k)=\operatorname{Ext}_{k G}^{*}(k, k)$ is a finitely generated $k$-algebra and $H^{*}(G, M)=\operatorname{Ext}_{k G}^{*}(k, M)$ is a finitely generated $H^{*}(G, k)$-module.

(4.3) For compact Lie groups, the following is a theorem of Venkov [1959].

TheOREM 4.3.1. Let $G$ be a compact Lie group and $k$ a Noetherian ring of coefficients. If $G \rightarrow \mathrm{U}(n)$ is a faithful unitary representation ${ }^{3}$ of $G$ then $H^{*}(B G ; k)$

\footnotetext{
${ }^{3}$ The Peter-Weyl theorem implies that every compact Lie group has a faithful unitary representation, so that this is not a restriction on $G$.
} 
is finitely generated as a module over the image of the Chern classes (3.3.1):

$$
H^{*}(B \mathrm{U}(n) ; k)=k\left[c_{1}, \ldots, c_{n}\right] \rightarrow H^{*}(B G ; k) .
$$

In particular, $H^{*}(B G ; k)$ is a finitely generated $k$-algebra. If $H$ is a closed subgroup of $G$ then $H^{*}(B H ; k)$ is a finitely generated module over the image of the restriction map (3.2) from $G$ to $H$.

(4.4) There is an interesting generalization of compact Lie groups which has been extensively investigated by Dwyer and Wilkerson, among others. A loop space is by definition a space $X$ together with another space $Y$ and a homotopy equivalence $X \simeq \Omega Y$ between $X$ and the space of pointed maps from $S^{1}$ to $Y$. If, furthermore, $H_{*}(X ; \mathbb{Z})$ is finitely generated as an abelian group (in other words, if each $H_{i}(X ; \mathbb{Z})$ is finitely generated, and only nonzero for a finite number of different values of $i$ ), so that $X$ looks homologically like a finite $\mathrm{CW}$-complex, then we say that $X$ is a finite loop space. For example, if $G$ is a compact Lie group then $G \simeq \Omega B G$ and $G$ is a finite loop space. For this reason, in general, the notation for the space $Y$ is $B X$, and it is called the classifying space of the loop space $X$. But in spite of the notation, the space $Y$ cannot be recovered from the space $X$, so naming $Y=B X$ is regarded as part of the structure of the finite loop space $X$. The following theorem of Dwyer and Wilkerson [1994] generalizes Venkov's Theorem 4.3.1.

THEOREM 4.4.1. If $X$ is a finite loop space, then for any field $k$, the algebra $H^{*}(B X ; k)$ is finitely generated.

A closely related concept is that of a $p$-compact group, which is by definition a loop space $X$ which is $\mathbb{F}_{p^{-}}$-complete in the sense of [Bousfield and Kan 1972] $]^{4}$, $\mathbb{F}_{p}$-finite in the sense that $H^{*}\left(X ; \mathbb{F}_{p}\right)$ is finite, and such that $\pi_{0} X$ is a finite $p$ group. The $\mathbb{F}_{p}$-completion of a finite loop space is an example of a $p$-compact group. The following theorem is also proved by Dwyer and Wilkerson [1994].

THEOREM 4.4.2. If $X$ is a p-compact group then $H^{*}\left(B X ; \mathbb{F}_{p}\right)$ is a finitely generated $\mathbb{F}_{p}$-algebra.

(4.5) For infinite discrete groups, the question of finite generation is more delicate, and there are various theorems for some special classes of infinite groups. For example, the cohomology of an arithmetic group with coefficients in $\mathbb{Z}$ or a field is finitely generated. More generally, we have the following theorem.

THEOREM 4.5.1. If a discrete group $G$ has a subgroup $H$ of finite index, such that there is a classifying space $B H$ which is a finite $C W$ complex, and $k$ is a Noetherian commutative ring of coefficients, then $H^{*}(G, k)$ is Noetherian.

\footnotetext{
${ }^{4}$ Bousfield-Kan $\mathbb{F}_{p}$-completion of a space is analogous to completion with respect to a prime ideal, inasmuch as it isolates the homotopy theoretic information at the prime $p$ and kills torsion coprime to $p$. It is used in order to do homotopy theory one prime at at time.
} 
Proof. We can take $H$ to be normal, and then the spectral sequence

$$
H^{*}\left(G / H, H^{*}(H, k)\right) \Longrightarrow H^{*}(G, k)
$$

has Noetherian $E_{2}$ page, and so $H^{*}(G, k)$ is Noetherian.

(4.6) A pro p-group is defined to be in inverse limit of finite $p$-groups. For pro $p$-groups, we have the following finite generation theorem of Minh and Symonds [2004].

TheOREM 4.6.1. Let $G$ be a pro p-group.

(i) The cohomology ring $H_{c}^{*}\left(G, \mathbb{F}_{p}\right)$ is finitely generated over $\mathbb{F}_{p}$ if and only if $G$ contains an open normal torsion-free subgroup $U$ such that $H_{c}^{*}\left(U, \mathbb{F}_{p}\right)$ is finite.

(ii) If $H_{c}^{*}\left(G, \mathbb{F}_{p}\right)$ is finitely generated then the number of conjugacy classes of finite subgroups of $G$ is finite.

(iii) $H_{c}^{*}\left(G, \mathbb{F}_{p}\right)$ modulo its nil radical is a finitely generated $\mathbb{F}_{p}$-algebra if and only if $G$ has only a finite number of conjugacy classes of finite elementary abelian p-subgroups.

\section{Krull Dimension}

(5.1) For a finitely generated graded commutative algebra $R$ over a field $k$, there are several ways to define Krull dimension, which all give the same answer.

(5.1.1) Noether normalization (2.5) guarantees the existence of a homogeneous polynomial ring $k\left[x_{1}, \ldots, x_{r}\right]$ over which $R$ is finitely generated as a module. The integer $r$ is the Krull dimension of $R$.

(5.1.2) If $\mathfrak{m}=\mathfrak{p}_{0} \supset \mathfrak{p}_{1} \supset \mathfrak{p}_{2} \supset \cdots \supset \mathfrak{p}_{r}$ is the longest chain of homogeneous prime ideals in $R$ and proper inclusions then $r$ is the Krull dimension of $R$.

(5.1.3) Set $p_{R}(t)=\sum_{i=0}^{\infty} t^{i} \operatorname{dim}_{k} R_{i}$. Then $p_{R}(t)$ is a rational function of $t$, and the order of the pole at $t=1$ is the Krull dimension of $R$.

(5.2) The first results on Krull dimension for cohomology rings are due to Quillen [1971b; 1971c], and are expressed in terms of the $p$-rank $r_{p}(G)$, where $p \geq 0$ is the characteristic of $k$. If $p$ is a prime, this is defined to be the largest $r$ such that $G$ has an elementary abelian subgroup of rank $r$; in other words, such that $(\mathbb{Z} / p)^{r} \subseteq G$. If $G$ is a compact Lie group, this is at least as big as the Lie rank $r_{0}(G)$, which is defined to be the rank $r_{0}$ of a maximal torus $\left(S^{1}\right)^{r_{0}} \subseteq G$. Quillen's theorem is as follows.

THEOREM 5.2.1. Let $G$ be a compact Lie group, and $k$ be a field of characteristic $p \geq 0$. Then the Krull dimension of $H^{*}(B G ; k)$ is equal to $r_{p}(G)$.

(5.3) In the special case where $G$ is finite, this means that the Krull dimension of $H^{*}(G, k)$ is equal to $r_{p}(G)$. Quillen [1971c] proved that the same holds more generally for groups of finite virtual cohomological dimension; in other words, 
for discrete groups $G$ containing a normal subgroup $H$ of finite index such that $H^{n}(H, M)=0$ for all large enough values of $n$ and all $\mathbb{Z} H$-modules $M$. The cohomology ring of $G$ is not necessarily finitely generated in this situation, but it is finitely generated modulo its nil radical.

In fact, in these cases, Quillen obtained much more than just the Krull dimension. He obtained a complete description of the maximal ideal spectrum of $H^{*}(B G ; k)$ up to inseparable isogeny, in terms of the Quillen category $\mathscr{A}_{p}(G)$ whose objects are the finite elementary abelian $p$-subgroups of $G$, and whose arrows are the monomorphisms induced by conjugation in $G$.

THEOREM 5.3.1. The restriction map (3.2)

$$
H^{*}(B G ; k) \rightarrow \lim _{E \in \mathscr{A}_{p}(G)} H^{*}(B E ; k)
$$

is an inseparable isogeny. In other words, the kernel of this map consists of nilpotent elements, and if $x$ is an element of the right hand side then $x^{p^{a}}$ is in the image for some $a \geq 0$.

We interpret Theorem 5.3.1 in terms of varieties in $\S 9$. But for now, notice that the cohomology of a finite elementary abelian $p$-group of rank $r$ is described in (2.2.2). In particular, modulo its nil radical it is always a polynomial ring in $r$ generators.

Corollary 5.3.3. The minimal primes in $H^{*}(B G ; k)$ are in one-one correspondence with the conjugacy classes of maximal elementary abelian p-subgroups of $G$, with respect to inclusion. If $E$ is a maximal elementary abelian p-subgroup, then the corresponding minimal prime is $\sqrt{\operatorname{ker}\left(\operatorname{res}_{G, E}\right)}$, the radical of the kernel of the restriction map (3.2) from $G$ to $E$. The Krull dimension of the quotient by this minimal prime is equal to the rank $r_{p}(E)$.

(5.4) The analog of the inseparable isogeny (5.3.2) was also proved by Quillen [1971c, Proposition 13.4] in the case of a profinite group with a finite number of conjugacy classes of elementary abelian $p$-subgroups.

\section{Depth}

(6.1) In contrast with Krull dimension, the depth of a cohomology ring is harder to compute. There are many interesting classes of groups for which the cohomology is known to be Cohen-Macaulay, even though this is less common for general finite groups, let alone for more general classes of groups.

(6.1.1) Groups with abelian Sylow $p$-subgroups have Cohen-Macaulay cohomology [Duflot 1981].

(6.1.2) $\quad \mathrm{GL}_{n}\left(\mathbb{F}_{q}\right)$ in characteristic not dividing $q$ [Quillen 1972], as well as various other finite groups of Lie type away from their defining characteristic, have 
Cohen-Macaulay cohomology (see [Fiedorowicz and Priddy 1978] for the classical groups and [Kleinerman 1982] for the groups of exceptional Lie type).

(6.1.3) $(p=2)$ Groups with almost extraspecial Sylow 2-subgroups ${ }^{5}$ have Cohen-Macaulay cohomology [Quillen 1971a].

(6.1.4) $(p=2)$ Finite simple groups of 2-rank at most three have Cohen-Macaulay cohomology [Adem and Milgram 1995].

(6.1.5) Finite groups of Lie type in the defining characteristic and finite symmetric groups almost never have Cohen-Macaulay cohomology, because they have maximal elementary abelian $p$-subgroups of different ranks, and hence minimal primes with quotients of different dimensions by Corollary 5.3.3.

(6.1.6) $(p=2)$ By computations of Carlson [ $\geq 2004]$, of the 267 isomorphism classes of 2-groups of order 64, 119 have Cohen-Macaulay cohomology rings. The depth differs from the Krull dimension by one in 126 cases and by two in the remaining 22 cases. See the Appendix at the end of these notes for more detailed information.

(6.2) As far as group theoretic characterizations of depth are concerned, the best theorems to date only give bounds on the depth. For example, Duflot's theorem [1981] gives the following lower bound.

TheOrem 6.2.1. Let $G$ be a finite group and $k$ a field of characteristic $p$. Then the depth of $H^{*}(G, k)$ is greater than or equal to the p-rank of the center of a Sylow p-subgroup $S$ of $G$. In particular, if $|G|$ is divisible by $p$ then $H^{*}(G, k)$ has strictly positive depth.

The bound of Theorem 6.2.1 gives the exact value for the depth for 193 of the 267 groups of order 64 .

(6.3) Theorem 6.2.1 generalizes to compact Lie groups as follows. If $G$ is a compact Lie group and $T$ is a maximal torus, then the inverse image $S \subseteq G$ of a Sylow $p$-subgroup of $N_{G}(T) / T$ is called a Sylow p-toral subgroup of $G$. The Sylow $p$-toral subgroups play the same role for compact Lie groups that Sylow $p$-subgroups do for finite groups. The crucial property as far as cohomology is concerned is that the restriction map $(3.2) H^{*}(B G ; k) \rightarrow H^{*}(B S ; k)$ followed by the transfer map $H^{*}(B S ; k) \rightarrow H^{*}(B G ; k)$ of [Becker and Gottlieb 1975] is the identity. ${ }^{6}$ Since the transfer map is a map of $H^{*}(B G ; k)$-modules, it follows that $H^{*}(B G ; k)$ is a direct summand of $H^{*}(B S ; k)$ as an $H^{*}(B G ; k)$-module. In particular, the depth of $H^{*}(B G ; k)$ is at least as big as the depth of $H^{*}(B S ; k)$.

\footnotetext{
${ }^{5} \mathrm{~A}$ finite $p$-group $P$ is said to be almost extraspecial if $P$ has a central subgroup $Z \cong \mathbb{Z} / p$ such that $P / Z$ is elementary abelian.

${ }^{6}$ For any closed subgroup, the composite of the restriction and the transfer is multiplication by the Euler characteristic of the quotient. The analog of the third Sylow theorem says that $\chi(G / S)$ is congruent to 1 modulo $p$.
} 
Stong (unpublished) showed how to generalize Duflot's proof to this situation, and showed that the depth of $H^{*}(B G ; k)$ is at least as big as $r_{p}(Z(S))$. Broto and Henn [1993] gave another proof which is conceptually easier, and goes as follows. We begin by establishing the notation.

If $C$ is a central elementary abelian $p$-subgroup of $S$, then the multiplication maps $C \times C \rightarrow C$ and $\mu: C \times S \rightarrow S$ are group homomorphisms. This means that $H^{*}(B C ; k)$ is a graded commutative and cocommutative Hopf algebra, $H^{*}(B S ; k)$ is an $H^{*}(B C ; k)$-comodule algebra via

$$
\mu^{*}: H^{*}(B S ; k) \rightarrow H^{*}(B C ; k) \otimes_{k} H^{*}(B S ; k),
$$

and $H^{*}(B G ; k)$ is a sub-comodule algebra.

Since $H^{*}(B C ; k)$ is Cohen-Macaulay $(2.2 .2)$, we can find elements $\zeta_{1}, \ldots, \zeta_{r} \in$ $H^{*}(B G ; k)$ whose restriction to $C$ form a homogeneous system of parameters and hence a regular sequence $x_{1}, \ldots, x_{r} \in H^{*}(B C ; k)$. One way to do this is to use Theorem 4.3.1 and throw away redundant Chern classes. We claim that $\zeta_{1}, \ldots, \zeta_{r}$ form a regular sequence in $H^{*}(B G ; k)$. Setting $\xi_{i}=\mu^{*}\left(\zeta_{i}\right)$, the elements $\xi_{1}, \ldots, \xi_{r}$ are elements of $H^{*}(B(C \times G) ; k) \cong H^{*}(B C ; k) \otimes_{k} H^{*}(B G ; k)$ (see $(2.2 .1)$ ) whose restrictions to the first factor are $x_{1}, \ldots, x_{r}$ and whose restrictions to the second factor are $\zeta_{1}, \ldots, \zeta_{r}$. So

$$
\mu^{*}\left(\zeta_{i}\right)=x_{i} \otimes 1+\cdots+1 \otimes \zeta_{i} .
$$

We begin with $\zeta_{1}$. If $y$ is a nonzero element in $H^{d}(B G ; k)$ such that $\zeta_{1} y=0$, then $\mu^{*}\left(\zeta_{1} y\right)=0$. Then $\mu^{*}(y)$ is a sum of tensors, and we separate out the terms whose degree in $H^{*}(B C ; k)$ are highest, say

$$
\mu^{*}(y)=\sum_{j} u_{j} \otimes v_{j}+\text { terms of lower first degree. }
$$

Then

$$
\mu^{*}\left(\zeta_{1} y\right)=\sum_{j} x_{1} u_{j} \otimes v_{j}+\text { terms of lower first degree. }
$$

But multiplication by $x_{1} \otimes 1$ is injective on $H^{*}(B C ; k) \otimes H^{*}(B G ; k)$, so the only way this can be zero is for $\sum_{j} u_{j} \otimes v_{j}$ to be zero. This means that $\mu^{*}(y)=0$ and so $y=0$.

The same argument works inductively, because the map $\mu^{*}$ passes down to a well defined map

$\mu^{*}: H^{*}(B G ; k) /\left(\zeta_{1}, \ldots, \zeta_{i}\right) \rightarrow H^{*}(B C ; k) /\left(x_{1}, \ldots, x_{i}\right) \otimes H^{*}(B G ; k) /\left(\zeta_{1}, \ldots, \zeta_{i}\right)$.

Applying the same argument to $\zeta_{i+1}$ using this map, we see that multiplication by $\zeta_{i+1}$ is injective on $H^{*}(B G ; k) /\left(\zeta_{1}, \ldots, \zeta_{i}\right)$. This inductive argument proves that $\zeta_{1}, \ldots, \zeta_{r}$ is a regular sequence in $H^{*}(B G ; k)$, and completes the BrotoHenn proof of the following version of Duflot's theorem. 
THEOREM 6.3.1. Let $G$ be a compact Lie group and $k$ a field of characteristic $p$. Then the depth of $H^{*}(B G ; k)$ is greater than or equal to the p-rank of the center of a Sylow p-toral subgroup $S$ of $G$.

(6.4) Green [2003] extended the above idea to prove a stronger result. An element $x \in H^{*}(B S ; k)$ is said to be primitive if its image under $\mu^{*}$ is equal to $1 \otimes x$. Since $\mu^{*}$ is a ring homomorphism, the primitives form a subring of $H^{*}(B S ; k)$. Since both $\mu$ and the projection onto the second factor of $C \times S$ have the same composite with the quotient map $S \rightarrow S / C$, it follows that the image of the inflation map (see $(3.2)) H^{*}(B(S / C) ; k) \rightarrow H^{*}(B S ; k)$ consists of primitive elements. If $I$ is an ideal in $H^{*}(B G ; k)$ generated by a regular sequence of primitive elements, then we can replace $H^{*}(B G ; k)$ by $H^{*}(B G ; k) / I$ in the above argument for Duflot's theorem, to obtain the following.

TheOREM 6.4.1. Let $G$ be a compact Lie group with Sylow p-toral subgroup $S$, and set $r=r_{p}(Z(S))$. If there is a regular sequence of length $s$ in $H^{*}(B G ; k)$ which consists of primitive elements, then the depth of $H^{*}(B G ; k)$ is at least $r+s$.

\section{Associated Primes and Steenrod Operations}

(7.1) Depth of the cohomology ring is closely linked with the action of the Steenrod operations (3.5). For example, an analysis of unstable algebras over the Steenrod algebra gives rise to a way of computing the depth with a single test sequence.

The test sequence takes the form of Dickson invariants. If $\mathbb{F}_{p}\left[x_{1}, \ldots, x_{r}\right]$ is a polynomial ring, then the general linear group $\mathrm{GL}_{r}\left(\mathbb{F}_{p}\right)$ acts by linear substitutions, and Dickson [1911] proved that the invariants form a polynomial ring

$$
\mathbb{F}_{p}\left[x_{1}, \ldots, x_{r}\right]^{\mathrm{GL}_{r}\left(\mathbb{F}_{p}\right)}=\mathbb{F}_{p}\left[c_{r, r-1}, \ldots, c_{r, 0}\right] .
$$

The Dickson invariant $c_{r, i}$ has degree $p^{r}-p^{i}$ in the variables $x_{1}, \ldots, x_{r}$. The Dickson invariants are further studied in [Wilkerson 1983], where the action of the Steenrod operations on them is also described.

If $G$ is a compact Lie group of $p$-rank $r$, then it is shown in [Benson and Wilkerson 1995] that it follows from Quillen's Theorem 5.3.1 that as an algebra over the Steenrod algebra, $H^{*}\left(B G ; \mathbb{F}_{p}\right)$ always contains a copy of the Dickson invariants as a homogeneous system of parameters, where, for a suitable integer $a \geq 0$ depending on $G, x_{1}, \ldots, x_{r}$ are taken to have degree $2^{a}$ if $p=2$ and $2 p^{a}$ if $p$ is odd. So

$$
\left|c_{r, i}\right|= \begin{cases}2^{a+r}-2^{a+i} & (p=2) \\ 2\left(p^{a+r}-p^{a+i}\right) & (p \text { odd }) .\end{cases}
$$

If $E$ is an elementary abelian $p$-subgroup of $G$ of rank $s \leq r$, then the restriction to $H^{*}(B E ; k)$ of $c_{r, i} \in H^{*}(B G ; k)$ is equal to $c_{s, i-r+s}^{p^{a+r-s}}$; thus it is a power of 
the Dickson invariant in the polynomial generators $(2.2 .2)$ in degree one $(p=2)$ or two ( $p$ odd) of $H^{*}(B E ; k)$.

The Landweber-Stong conjecture, proved by Bourguiba and Zarati [1997], implies the following.

THEOREM 7.1.1. The depth of $H^{*}\left(B G ; \mathbb{F}_{p}\right)$ is equal to the maximum value of $d$ for which $c_{r, r-1}, \ldots, c_{r, r-d}$ is a regular sequence.

Theorem 8.1.8 of [Neusel 2000] proves the much stronger statement that such a copy of the Dickson algebra can be found in any Noetherian unstable algebra over the Steenrod algebra. The conclusion of Theorem 7.1.1 holds in this more general context. The proof given by Bourguiba and Zarati makes heavy use of the machinery of unstable algebras over the Steenrod algebra. A proof without this machinery, but which only works in the context of the cohomology of a finite group, can be found in [Benson 2004].

The Dickson invariants have further desirable properties among all homogeneous systems of parameters in cohomology. For example, if $H$ is a closed subgroup of $G$ of $p$-rank $s$ then the restrictions of $c_{r, r-1}, \ldots, c_{r, r-s}$ are Dickson invariants forming a homogeneous system of parameters in $H^{*}(B H ; k)$. Furthermore, $c_{r, i}$ is a sum of transfers from centralizers of elementary abelian subgroups of rank $i$.

(7.2) To get further with depth, it is necessary to get some understanding of the associated primes in group cohomology. In general, the dimension of the quotient by an associated prime is an upper bound for the depth. For general commutative rings, the depth cannot be computed from the dimensions of the quotients by associated primes, but in the case of cohomology of a finite group, we have the following conjecture of Carlson [1999]. Partial results on this conjecture have been obtained by Green [2003].

Conjecture 7.2.1. Let $G$ be a finite group and $k$ a field. Then $H^{*}(G, k)$ has an associated prime $\mathfrak{p}$ such that the Krull dimension of $H^{*}(G, k) / \mathfrak{p}$ is equal to the depth of the $H^{*}(G, k)$.

(7.3) The following theorem of Wilkerson [1982] shows that associated primes are invariant under the action of the Steenrod operations. Since it is not easy to find an explicit reference, we include a complete proof here.

THEOREM 7.3.1. Let $H$ be a graded commutative unstable algebra over the Steenrod algebra. For example, these hypotheses are satisfied if $H$ is the mod $p$ cohomology ring of a space, see (3.5). Then the radical of the annihilator of any element is invariant under the action of the Steenrod operations.

More explicitly, for $p=2$, if $y$ annihilates $x$ and $2^{n}>|x|$ then $\left(\mathrm{Sq}^{k} y\right)^{2^{n}} x=0$ for all $k>0$. For $p$ odd, if $y$ annihilates $x$ and $p^{n}>2(p-1)|x|$ then $\left(P^{k} y\right)^{p^{n}}=0$ for all $k>0$. 
Proof. We give the proof for $p=2$; the proof for $p$ odd is the same, with $P^{i}$ instead of $\mathrm{Sq}^{i}$ and $p^{n}$ instead of $2^{n}$.

Let $x \in H$, let $I$ be the annihilator of $x$, and let $y \in I$. We have $y^{2^{n}} x=0$, and so for any $k>0$ we have $\operatorname{Sq}^{2^{n} k}\left(y^{2^{n}} x\right)=0$. Using the Cartan formula (3.5.2) we obtain

$$
\sum_{i=0}^{2^{n} k} \mathrm{Sq}^{2^{n} k-i}\left(y^{2^{n}}\right) \mathrm{Sq}^{i} x=0
$$

The Cartan formula and divisibility properties of binomial coefficients imply that $\mathrm{Sq}^{2^{n} k-i}\left(y^{2^{n}}\right)=0$ unless $i$ is of the form $2^{n} j$, and in that case we have $\mathrm{Sq}^{2^{n}(k-j)}\left(y^{2^{n}}\right)=\left(\mathrm{Sq}^{k-j} y\right)^{2^{n}}$. So the above equation becomes

$$
\sum_{j=0}^{k}\left(\mathrm{Sq}^{k-j} y\right)^{2^{n}} \mathrm{Sq}^{2^{n} j} x=0 .
$$

Since $2^{n}>|x|$, the unstable condition implies that the only term which survives in this sum is the term with $j=0$. So we have $\left(\mathrm{Sq}^{k} y\right)^{2^{n}} x=0$.

Since associated primes are annihilators, we get the following.

COROLLARY 7.3.2. The associated primes in a mod $p$ cohomology ring of a space are invariant under the Steenrod operations.

(7.4) The importance of the Steenrod invariance of the associated primes in the cohomology of groups comes from the following theorem of Serre [1965b, Proposition 1].

TheOREm 7.4.1. Let $E$ be an elementary abelian p-group. The Steenrod invariant prime ideals in $H^{*}\left(E, \mathbb{F}_{p}\right)$ are in one-one correspondence with the subgroups of $E$. If $E^{\prime}$ is a subgroup of $E$ then the corresponding Steenrod invariant prime ideal is $\sqrt{\operatorname{ker}\left(\operatorname{res}_{E, E^{\prime}}\right)}$, the radical of the kernel of restriction from $E$ to $E^{\prime}$.

(7.5) Combining Theorem 7.4.1 with Quillen's Theorem 5.3.1, it follows that for any of the classes of groups for which that theorem holds, the Steenrod invariant primes in the mod $p$ cohomology ring are the radicals of the kernels of restriction to elementary abelian subgroups. So using Corollary 7.3.2 we have the following.

Theorem 7.5.1. Let $G$ be a compact Lie group. Then the Steenrod invariant prime ideals in $H^{*}\left(B G ; \mathbb{F}_{p}\right)$ are the ideals of the form $\sqrt{{\operatorname{ker}\left(\operatorname{res}_{G, E}\right)}_{1}}$, where $E$ is an elementary abelian p-subgroup of $G$. In particular, the associated primes are of this form.

(7.6) The corresponding result holds for the cohomology of groups of finite virtual cohomological dimension, and continuous cohomology of profinite groups with a finite number of conjugacy classes of elementary abelian $p$-subgroups.

(7.7) The question of exactly which elementary abelian subgroups give the associated primes is difficult. In the next section, we relate this to the question of finding upper bounds for the depth. 


\section{Associated Primes and Transfer}

(8.1) Upper bounds on the depth of the cohomology ring of a finite group come from a careful analysis of transfer and its relationship to the associated primes. If $H$ is a subgroup of a finite group $G$ and $M$ is a $k G$-module, then the transfer is a map

$$
\operatorname{Tr}_{H, G}: H^{n}(H, M) \rightarrow H^{n}(G, M) .
$$

It is defined by choosing a set of left coset representatives $g_{i}$ of $H$ in $G$. Let $P_{*}$ be a projective resolution of $k$ as a $k G$-module. Given a representative cocycle, which is a $k H$-module homomorphism $\hat{\zeta}: P_{n} \rightarrow M$, the transfer $\operatorname{Tr}_{H, G}(\zeta)$ is represented by $\sum_{i} g_{i}(\hat{\zeta})$, which is a $k G$-module homomorphism.

The reason why the transfer map is relevant is that if $H$ is a subgroup of $G$ and $M$ is a $k G$-module, then $\operatorname{Tr}_{H, G}$ is $H^{*}(G, k)$-linear, when $H^{*}(H, M)$ is viewed as a right module over $H^{*}(G, k)$ via the restriction map $H^{*}(G, k) \rightarrow H^{*}(H, k)$. In other words, the following identity holds. If $\zeta \in H^{*}(G, k)$ and $\eta \in H^{*}(H, M)$ then

$$
\operatorname{Tr}_{H, G}\left(\eta \cdot \operatorname{res}_{G, H}(\zeta)\right)=\operatorname{Tr}_{H, G}(\eta) \cdot \zeta .
$$

In particular, if $\eta$ annihilates $\operatorname{res}_{G, H}(\zeta)$ then $\operatorname{Tr}_{H, G}(\eta)$ annihilates $\zeta$. For example, if $\zeta$ restricts to zero on some set of subgroups, then all transfers from those subgroups annihilate $\zeta$.

(8.2) One way to exploit the above observation is to use the following transfer theorem from [Benson 1993]. This generalizes a theorem of Carlson [1987] relating transfers from all proper subgroups of a $p$-group to the kernel of restriction to the center.

TheOREM 8.2.1. Suppose that $G$ is a finite group, and $k$ is a field of characteristic $p$. Let $\mathscr{H}$ be a collection of subgroups of $G$. Let $\mathscr{K}$ denote the collection of all elementary abelian p-subgroups $K$ of $G$ with the property that the Sylow $p$-subgroups of the centralizer $C_{G}(K)$ are not conjugate to a subgroup of any of the groups in $\mathscr{H}$.

Let $J$ be the sum of the images of transfer from subgroups in $\mathscr{H}$, which is an ideal in $H^{*}(G, k)$ by (8.1.1). Let $J^{\prime}$ be the intersection of the kernels of restriction to subgroups in $\mathscr{K}$, which is again an ideal in $H^{*}(G, k)$ (in case $\mathscr{K}$ is empty, this intersection is taken to be the ideal of all elements of positive degree). Then $J$ and $J^{\prime}$ have the same radical, $\sqrt{J}=\sqrt{J^{\prime}}$.

(8.3) Theorem 8.2.1 is the main ingredient in the proof of the following theorem of Carlson [1995] relating the associated primes with detection on centralizers.

TheOrem 8.3.1. Let $G$ be a finite group. Suppose that $H^{*}(G, k)$ has a nonzero element $\zeta$ which restricts to zero on $C_{G}(E)$ for each elementary abelian psubgroup $E \leq G$ of rank s. Then $H^{*}(G, k)$ has an associated prime $\mathfrak{p}$ such 
that the Krull dimension of $H^{*}(G, k) / \mathfrak{p}$ is strictly less than s. In particular, the depth of $H^{*}(G, k)$ is strictly less than $s$.

Proof. In Theorem 8.2.1, we take $\mathscr{H}$ to be the set of centralizers of elementary abelian $p$-subgroups of rank $s$. Then the elementary abelian $p$-subgroups in $\mathscr{K}$ have rank strictly less than $s$. So the theorem implies that the ideal $J$ has dimension strictly less than $s$.

If $\zeta$ is an element of $H^{*}(G, k)$ which restricts to zero on every element of $\mathscr{H}$, then by (8.1.1), $\zeta$ is annihilated by all transfers from $\mathscr{H}$. In other words, the annihilator of $\zeta$ contains $J$. Since the associated primes are the maximal annihilators, there is an associated prime containing $J$, and such an associated prime has dimension strictly less than $s$.

(8.4) Another way of stating the conclusion to the theorem above is that if $H^{*}(G, k)$ has depth at least $s$ then cohomology is detected on centralizers of rank $s$ elementary abelian $p$-sugroups of $G$.

\section{Idempotent Modules and Varieties}

(9.1) There is a method for systematically exploiting the connections between representation theory and cohomology, which was first introduced by Carlson [1981a; 1981b; 1983] for finitely generated modules, and by Benson, Carlson and Rickard [Benson et al. 1995; 1996] for infinitely generated modules.

Let $G$ be a finite group, and let $k$ be an algebraically closed field of characteristic $p$. We write $V_{G}$ for the maximal ideal spectrum of $H^{*}(G, k)$. This is a closed homogeneous affine variety. For example, if $G \cong(\mathbb{Z} / p)^{r}$ then $V_{G}=\mathbb{A}^{r}(k)$, affine $r$-space over $k$. Quillen's Theorem 5.3 .1 can be interpreted as saying that for any finite group $G$, the natural map

$$
\lim _{E \in \mathscr{A}_{G}} V_{E} \rightarrow V_{G}
$$

is bijective at the level of sets of points. However, it is usually not invertible in the category of varieties.

If $M$ is a finitely generated $k G$-module, then the kernel of the natural map

$$
H^{*}(G, k)=\operatorname{Ext}_{k G}^{*}(k, k) \stackrel{M \otimes_{k}-}{\longrightarrow} \operatorname{Ext}_{k G}^{*}(M, M)
$$

is an ideal in $H^{*}(G, k)$, which defines a closed homogeneous subvariety $V_{G}(M)$ of $V_{G}$. The same subvariety can be obtained by taking the intersection of the annihilators of $\operatorname{Ext}_{k G}^{*}(S, M)$ as $S$ runs over the simple $k G$-modules. Properties of varieties for modules include (9.1.1)-(9.1.5) below.

$$
\begin{aligned}
& V_{G}(M)=\{0\} \text { if and only if } M \text { is projective, } \\
& V_{G}(M \oplus N)=V_{G}(M) \cup V_{G}(N),
\end{aligned}
$$


(9.1.3) $\quad V_{G}(M \otimes N)=V_{G}(M) \cap V_{G}(N),^{7}$

(9.1.4) If $0 \neq \zeta \in H^{n}(G, k)$ is represented by a cocycle $\hat{\zeta}: \Omega^{n}(k) \rightarrow k$, let $L_{\zeta}$ be the kernel of $\hat{\zeta}$. Then $V_{G}\left(L_{\zeta}\right)$ is the hypersurface in $V_{G}$ determined by regarding $\zeta$ as an element of the coordinate ring of $V_{G}$.

(9.1.5) If $V$ is any closed homogeneous subvariety of $V_{G}$, then we can choose homogeneous elements $\zeta_{1}, \ldots, \zeta_{t} \in H^{*}(G, k)$ so that the intersection of the hypersurfaces they define is equal to $V$. Properties (9.1.3) and (9.1.4) then imply that

$$
V_{G}\left(L_{\zeta_{1}} \otimes \cdots \otimes L_{\zeta_{t}}\right)=V
$$

So every closed homogeneous subvariety is the variety of some module.

(9.2) For infinite dimensional modules, ${ }^{8}$ the definitions are more difficult. A tentative definition was given in [Benson et al. 1995], and the definition was modified in [Benson et al. 1996] to remedy some defects. We begin with some background on the stable module category. We write $\operatorname{Mod}(k G)$ for the category of $k G$-modules and module homomorphisms. The stable module category $\operatorname{StMod}(k G)$ has the same objects as $\operatorname{Mod}(k G)$, but the morphisms are

$$
\underline{\operatorname{Hom}}_{k G}(M, N)=\operatorname{Hom}_{k G}(M, N) / \operatorname{PHom}_{k G}(M, N)
$$

where $\operatorname{PHom}_{k G}(M, N)$ is the subspace consisting of maps which factor through some projective $k G$-module. One of the advantages of $\operatorname{StMod}(k G) \operatorname{over} \operatorname{Mod}(k G)$ is that if we define $\Omega M$ to be the kernel of a surjection from a projective module $P$ onto $M$, then $\Omega$ is a functor on $\operatorname{StMod}(k G)$. Since, over $k G$, projective modules are the same as injective modules, $\Omega$ is a self-equivalence of $\operatorname{StMod}(k G)$. Its inverse $\Omega^{-1}$ is defined by embedding $M$ into an injective $k G$-module $I$ and writing $\Omega^{-1} M$ for the quotient $I / M$.

The category $\operatorname{Mod}(k G)$ is abelian, but $\operatorname{StMod}(k G)$ is not. Instead it is a triangulated category. The triangles are of the form

$$
A \rightarrow B \rightarrow C \rightarrow \Omega^{-1} A
$$

where $0 \rightarrow A \rightarrow B \rightarrow C \rightarrow 0$ is a short exact sequence in $\operatorname{Mod}(k G)$. We write $\bmod (k G)$ and $\operatorname{stmod}(k G)$ for the full subcategories of finitely generated modules.

Write Proj $H^{*}(G, k)$ for the set of closed homogeneous irreducible subvarieties of $V_{G}$, and let $\mathscr{V}$ be a subset of Proj $H^{*}(G, k)$ which is closed under specialization,

\footnotetext{
${ }^{7}$ When we write $M \otimes N$ for $k G$-modules $M$ and $N$, we mean $M \otimes_{k} N$ with diagonal $G$ action. So an element $g \in G$ acts via $g(m \otimes n)=g m \otimes g n$. But the general element of $k G$ does not act in this fashion; rather, we extend linearly from the action of the group elements. See $\S 2.11$ of [Sri].

${ }^{8}$ The reason for interest in infinite dimensional modules in this context is similar to the reason for the interest in infinite $\mathrm{CW}$ complexes in algebraic topology. Namely, the representing objects for functors often turn out to be infinite. For example in algebraic topology, Eilenberg-Mac Lane spaces are the representing objects for cohomology, $B \mathrm{U}$ for K-theory, $M \mathrm{U}$ for cobordism, and so on.
} 
in the sense that if $V \in \mathscr{V}$ and $W \subseteq V$ then $W \in \mathscr{V}$. Let $\mathscr{M}$ be the full subcategory of $\operatorname{stmod}(k G)$ consisting of finitely generated modules $M$ such that $V_{G}(M)$ is a finite union of elements of $\mathscr{V}$. Then $\mathscr{M}$ is a thick subcategory of $\operatorname{stmod}(k G)$; in other words it is a full triangulated subcategory of $\operatorname{stmod}(k G)$ with the same definition of triangles, and is closed under taking direct summands. Furthermore, a tensor product of any module with a module in $\mathscr{M}$ gives an answer in $\mathscr{M}$, so we say that $\mathscr{M}$ is a tensor closed thick subcategory. To such a subcategory of $\operatorname{stmod}(k G)$, Rickard [1997] associates two idempotent modules ${ }^{9}$ $E_{\mathscr{V}}$ and $F_{\mathscr{V}}$ and a triangle

$$
E_{\mathscr{V}} \rightarrow k \rightarrow F_{\mathscr{V}} \rightarrow \Omega^{-1} E_{\mathscr{V}}
$$

in $\operatorname{StMod}(k G)$. This triangle is characterized by the statement that $E_{\mathscr{V}}$ can be written as a filtered colimit of modules in $\mathscr{M}$, and for any $M$ in $\mathscr{M}$, we have $\underline{\operatorname{Hom}}_{k G}\left(M, F_{\mathscr{V}}\right)=0$. This construction is the analog in representation theory of Bousfield localization [Bousfield 1979] in algebraic topology.

As an example, if $\zeta \in H^{n}(G, k)$ defines a hypersurface $V$ in $V_{G}$ and $\mathscr{V}$ is the set of subvarieties of $V$ then we write $E_{\zeta}$ and $F_{\zeta}$ instead of $E_{\mathscr{V}}$ and $F_{\mathscr{V}}$. If $\zeta$ is represented by a cocycle $\hat{\zeta}: \Omega^{n}(k) \rightarrow k$, then the module $F_{\zeta}$ can be constructed as follows. We can dimension shift $\hat{\zeta}$ to give maps

$$
k \stackrel{\Omega^{-n} \hat{\zeta}}{\longrightarrow} \Omega^{-n}(k) \stackrel{\Omega^{-2 n} \hat{\zeta}}{\longrightarrow} \Omega^{-2 n}(k) \stackrel{\Omega^{-3 n} \hat{\zeta}}{\longrightarrow} \cdots
$$

and the colimit is $F_{\zeta}$. So for example the cohomology of $F_{\zeta}$,

$$
\hat{H}^{*}\left(G, F_{\zeta}\right) \cong \hat{H}^{*}(G, k)_{\zeta} \cong H^{*}(G, k)_{\zeta}
$$

is the localization of either Tate or ordinary cohomology with respect to $\zeta$.

If we take the map from the first term in the sequence to the colimit and complete to a triangle, we get the module $E_{\zeta}$ and the triangle

$$
E_{\zeta} \rightarrow k \rightarrow F_{\zeta} \rightarrow \Omega^{-1} E_{\zeta}
$$

If $L_{\zeta^{i}}$ is the kernel of $\hat{\zeta}^{i}$ then $E_{\zeta}$ can be written as the colimit of

$$
\Omega^{-n} L_{\zeta} \rightarrow \Omega^{-2 n} L_{\zeta^{2}} \rightarrow \Omega^{-3 n} L_{\zeta^{3}} \rightarrow \cdots
$$

More generally, if $V$ is a closed homogeneous subvariety of $V_{G}$ defined by the vanishing of elements $\zeta_{1}, \ldots, \zeta_{t} \in H^{*}(G, k)$ and $\mathscr{V}$ is the set of subvarieties of $V$, we write $E_{V}$ and $F_{V}$ for $E_{\mathscr{V}}$ and $F_{\mathscr{V}}$. In this case, $E_{V}$ can be obtained as $E_{\zeta_{1}} \otimes \cdots \otimes E_{\zeta_{t}}$, and $F_{V}$ can be obtained by completing the map $E_{V} \rightarrow k$ to a triangle.

\footnotetext{
${ }^{9} \mathrm{~A}$ module $M$ is said to be idempotent if $M \otimes M$ is isomorphic to $M$ in $\operatorname{StMod}(k G)$. The only finite dimensional idempotent module is the trivial $k G$-module $k$.
} 
Now if $V$ is a closed homogeneous irreducible subvariety of $V_{G}$, let $\mathscr{W}$ be the set of subvarieties of $V_{G}$ which do not contain $V$. Then we define

$$
\kappa_{V}=E_{V} \otimes F_{\mathscr{W}}
$$

This is an idempotent module which corresponds to a layer of $\operatorname{stmod}(k G)$ consisting of modules with variety exactly $V$.

If $M$ is a $k G$-module, not necessarily finitely generated, we associate to $M$ a collection of varieties

$$
\mathscr{V}_{G}(M)=\left\{V \mid M \otimes \kappa_{V} \text { is not projective }\right\} \subseteq \operatorname{Proj} H^{*}(G, k)
$$

If $M$ happens to be finitely generated, then $\mathscr{V}_{G}(M)$ is just the collection of all subvarieties of $V_{G}(M)$. But for infinitely generated modules, the collection is not necessarily closed under specialization. The properties of $\mathscr{V}_{G}(M)$ include:

$$
\begin{aligned}
& \mathscr{V}_{G}(M)=\varnothing \text { if and only if } M \text { is projective, } \\
& \mathscr{V}_{G}\left(\bigoplus_{\alpha} M_{\alpha}\right)=\bigcup_{\alpha} \mathscr{V}_{G}\left(M_{\alpha}\right), \\
& \mathscr{V}_{G}(M \otimes N)=\mathscr{V}_{G}(M) \cap \mathscr{V}_{G}(N), \\
& \mathscr{V}_{G}\left(\kappa_{V}\right)=\{V\} .
\end{aligned}
$$

(9.2.7) It follows from (9.2.4) and (9.2.6) that every subset of Proj $H^{*}(G, k)$ occurs as $\mathscr{V}_{G}(M)$ for some $M$.

\section{Modules with Injective Cohomology}

In this section, we continue with our assumption that $G$ is a finite group and $k$ is a field.

(10.1) A better understanding of the modules $\kappa_{V}$ comes from understanding modules whose Tate cohomology is injective as a module over the Tate cohomology of the group. In this section, we shall see that there is an essentially unique module with a given injective as its cohomology [Benson and Krause 2002]. Conjecturally, these are the translates of the modules $\kappa_{V}$ described in the last section. This has been proved under some restrictive hypotheses in [Benson 2001], but at least it is true for elementary abelian groups, an important special case. The connection between the $\kappa_{V}$ and modules with injective cohomology involves the study of the local cohomology of the cohomology ring, $H_{\mathfrak{p}}^{* *} H^{*}(G, k)$. This is the subject of the next section.

(10.2) It is well known that the indecomposable injective modules over a commutative Noetherian ring $R$ are precisely the injective hulls $E(R / \mathfrak{p})$ of the modules $R / \mathfrak{p}$, as $\mathfrak{p}$ ranges over the prime ideals of $R$, and that a general injective 
module can be written in an essentially unique way as a direct sum of indecomposable injectives. ${ }^{10}$ For a Noetherian graded commutative ring, the classification of injective graded modules is the same, except that we must restrict our attention to homogeneous prime ideals, and we must allow degree shift. If $\mathfrak{p}$ is a homogeneous prime ideal in $H^{*}(G, k)$ and $d$ is an integer, we define $I_{\mathfrak{p}}$ to be $E\left(H^{*}(G, k) / \mathfrak{p}\right)$ and $I_{\mathfrak{p}}[n]$ to be the result of shifting degrees by $n$. The notation here is that a shift of $[n]$ in a graded module means that the degree $d$ part of the shifted module is the same as the degree $(n+d)$ part of the original module.

(10.3) Recall that the ordinary cohomology $H^{*}(G, k)$ is Noetherian, whereas Tate cohomology $\hat{H}^{*}(G, k)$ is usually not. The way to get from injective modules over ordinary cohomology to injectives over Tate cohomology is by coinduction. If $I$ is an injective $H^{*}(G, k)$-module, we define $\hat{I}$ to be the injective $\hat{H}^{*}(G, k)$ module

$$
\hat{I}=\operatorname{Hom}_{H^{*}(G, k)}^{*}\left(\hat{H}^{*}(G, k), I\right) .
$$

The notation here is that $\mathrm{Hom}^{n}$ denotes the graded homomorphisms which increase degree by $n$. If there is no superscript, it is assumed that $n=0$.

TheOrem 10.3.1. Every injective $\hat{H}^{*}(G, k)$-module is of the form $\hat{I}$ for some injective $H^{*}(G, k)$-module $I$.

(10.4) In order to understand what happens when we coinduce $I=I_{\mathfrak{p}}[n]$, we consider two cases. Let $\mathfrak{m}=H^{+}(G, k)$ be the maximal ideal of positive degree elements in $H^{*}(G, k)$. If $\mathfrak{p}$ is not equal to $\mathfrak{m}$ then $\hat{I}=I$. More precisely, the restriction of $\hat{I}$ to an $H^{*}(G, k)$-module is naturally isomorphic to $I$. On the other hand, if $\mathfrak{p}=\mathfrak{m}$ then Tate duality (3.6.1) says that $I=\hat{H}^{-}(G, k)[n-1]$. In this case, $\hat{I}=\hat{H}^{*}(G, k)[n-1]$.

Since $\hat{H}^{*}(G, k)$ is usually not Noetherian, coinduction does not preserve direct sums. Actually, it does preserve direct sums as long as the injective only has copies of $I_{\mathfrak{p}}[n]$ with $\mathfrak{p} \neq \mathfrak{m}$, and no copies of $I_{\mathfrak{m}}[n]$; in other words if $\operatorname{Hom}_{H^{*}(G, k)}^{*}(k, I)=0$. Although $\hat{H}^{*}(G, k)$ is not Noetherian, it is shown in [Benson and Krause 2002] that the general injective $\hat{H}^{*}(G, k)$-module has the form

$$
\bigoplus \widehat{I_{\mathfrak{p}}[n]} \oplus \quad E\left(\bigoplus \hat{H}^{*}(G, k)[n-1]\right)
$$

where $\mathfrak{p} \neq \mathfrak{m}$ for each of the left hand summands, and $E(-)$ stands for injective hull over $\hat{H}^{*}(G, k)$.

A way to construct modules with injective cohomology is to use the Brown representability theorem [Brown 1965; Neeman 1996]. If $I$ is an injective $H^{*}(G, k)$ module, the functor from $\operatorname{StMod}(k G)$ to vector spaces, which takes a $k G$-module

\footnotetext{
${ }^{10} \mathrm{~A}$ ring $R$ is Noetherian if and only if an arbitrary direct sum of injective $R$-modules is injective.
} 
$M$ to the degree preserving homomorphisms

$$
\operatorname{Hom}_{H^{*}(G, k)}\left(\hat{H}^{*}(G, M), I\right)
$$

is exact (in other words it takes triangles in $\operatorname{StMod}(k G)$ to long exact sequences) and takes direct sums to direct products. Brown's representability theorem says that any such functor is representable. In other words, there exists a $k G$-module $T(I)$ and a functorial isomorphism

$$
\operatorname{Hom}_{H^{*}(G, k)}\left(\hat{H}^{*}(G, M), I\right) \cong \underline{\operatorname{Hom}}_{k G}(M, T(I)) .
$$

We remark that we could just as easily have replaced the left hand side of this isomorphism with $\operatorname{Hom}_{\hat{H}^{*}(G, k)}\left(\hat{H}^{*}(G, M), \hat{I}\right)$, because this gives the same answer, and because by Theorem 10.3.1, every injective $\hat{H}^{*}(G, k)$-module is coinduced from $H^{*}(G, k)$.

(10.5) Yoneda's lemma says that all natural transformations from a representable functor are representable. Applying this to natural transformations arising from homomorphisms between injective $H^{*}(G, k)$-modules, this makes $T$ into a functor from the full subcategory $\operatorname{Inj} H^{*}(G, k)$ of injective $H^{*}(G, k)$ modules to the stable module category $\operatorname{StMod}(k G)$. Here are some properties of the modules $T(I)$, proved in [Benson and Krause 2002].

(10.5.1) $T\left(I_{\mathfrak{m}}[n]\right) \cong \Omega^{-n+1}(k)$.

(10.5.2) If $\mathfrak{p} \neq \mathfrak{m}$ then $T\left(I_{\mathfrak{p}}[n]\right)$ is an infinitely generated module whose variety is given by $\mathscr{V}_{G}\left(T\left(I_{\mathfrak{p}}[n]\right)\right)=\{V\}$, where $V$ is the irreducible variety corresponding to $\mathfrak{p}$.

(10.5.3) $\operatorname{Hom}_{H^{*}(G, k)}^{*}\left(\hat{H}^{*}(G, M), I\right) \cong \widehat{\operatorname{Ext}}_{k G}^{*}(M, T(I))$. This isomorphism follows by dimension shifting the defining isomorphism (10.4.2).

(10.5.4) $\quad \hat{H}^{*}(G, T(I)) \cong \hat{I}$. This is the special case of (iii) where $M=k$.

(10.5.5) If $\mathfrak{p} \neq \mathfrak{m}$ then $\widehat{\operatorname{Ext}}_{k G}^{*}\left(T\left(I_{\mathfrak{p}}[n]\right), T\left(I_{\mathfrak{p}}[n]\right)\right) \cong \operatorname{End}_{H^{*}(G, k)}^{*}\left(I_{\mathfrak{p}}\right)$. By a theorem of Matlis [1958], $\operatorname{End}_{H^{*}(G, k)}^{*}\left(I_{\mathfrak{p}}\right)$ is isomorphic to the $\mathfrak{p}$-adic completion of cohomology,

$$
H^{*}(G, k)_{\mathfrak{p}}^{\wedge}=\underset{n}{\lim _{n}} H^{*}(G, k)_{\mathfrak{p}} / \mathfrak{p}_{\mathfrak{p}}^{n}
$$

More generally, if $I$ has no copies of $I_{\mathfrak{m}}[n]$ as summands, or equivalently, if there are no homomorphisms from any degree shifted copy of $k$ to $I$, then

$$
\underline{\operatorname{Hom}}_{k G}\left(T(I), T\left(I^{\prime}\right)\right) \cong \operatorname{Hom}_{H^{*}(G, k)}\left(I, I^{\prime}\right)
$$

So $T$ is a fully faithful functor on the full subcategory $\ln _{0} H^{*}(G, k)$ of injective modules $I$ satisfying $\operatorname{Hom}_{H^{*}(G, k)}^{*}(k, I)=0$. 
(10.5.6) The modules $T(I)$ are pure injective. This means that if a short exact sequence of $k G$-modules

$$
0 \rightarrow T(I) \rightarrow X \rightarrow Y \rightarrow 0
$$

has the property that every morphism from any finitely generated module to $Y$ lifts to $X$, then it splits. The reason for this is that $T(I)$ is a direct summand of a direct product of finitely generated $k G$-modules. In fact,

(10.5.7) The modules $T(I)$ are precisely the direct summands of direct products of modules isomorphic to $\Omega^{n}(k)$ for $n \in \mathbb{Z}$. The indecomposable ones are exactly $T\left(I_{\mathfrak{p}}[n]\right)$. So we obtain an embedding of Proj $H^{*}(G, k)$ into the Ziegler spectrum [Ziegler 1984] of pure injective $k G$-modules with the Zariski topology (modulo the translation $\Omega$ ), and we can recover $\operatorname{Proj} H^{*}(G, k)$ from the category $\operatorname{StMod}(k G)$ if we know where the translates of the trivial module are.

(10.6) The following conjecture from [Benson 2001] relates the modules $T\left(I_{\mathfrak{p}}[n]\right)$ described in this section and the modules $\kappa_{V}$ described in the previous section.

CONJECTURE 10.6.1. If $\mathfrak{p}$ is the homogeneous prime ideal corresponding to a closed homogeneous irreducible subvariety $V$ of dimension $d$ in $V_{G}$, then there is an isomorphism $T\left(I_{\mathfrak{p}}[n]\right) \cong \Omega^{-n-d} \kappa_{V}$ in $\operatorname{StMod}(k G)$.

This conjecture is known to hold if $H^{*}(G, k)_{\mathfrak{p}}$ is Cohen-Macaulay. In the next few sections, we describe how to view this conjecture in terms of local cohomology and Grothendieck's local duality. See Conjecture 13.2.2.

(10.7) Another conjecture, related in philosophy to Conjecture 10.6.1, comes from an idea of Amnon Neeman. If $M$ is a $k G$-module, not necessarily finitely generated, then for each simple module $S$, consider a minimal injective resolution of $\widehat{\operatorname{Ext}}_{k G}^{*}(S, M)$ as a module over $\hat{H}^{*}(G, k)$,

$$
0 \rightarrow \widehat{\operatorname{Ext}}_{k G}^{*}(S, M) \rightarrow \hat{I}_{0} \rightarrow \hat{I}_{1} \rightarrow \hat{I}_{2} \rightarrow \cdots
$$

Each injective in such a resolution can be written in the form (10.4.1), and we can ask which nonmaximal prime ideals occur in such a decomposition. Since each $\hat{I}_{j}$ is coinduced from some injective $H^{*}(G, k)$-module $I_{j}$, we have

$$
\operatorname{Hom}_{\hat{H}^{*}(G, k)}^{*}\left(H^{*}(G, k) / \mathfrak{p}, \hat{I}_{j}\right) \cong \operatorname{Hom}_{H^{*}(G, k)}^{*}\left(H^{*}(G, k) / \mathfrak{p}, I_{j}\right),
$$

and this is nonzero exactly for the primes appearing in this minimal resolution. Since coinduction is exact on injectives away from the maximal ideal, it does not matter whether we resolve over $\hat{H}^{*}(G, k)$ or $H^{*}(G, k)$. The following conjecture says that the primes appearing in these minimal resolutions, as $S$ runs over the simple $k G$-modules, correspond exactly to the varieties in $\mathscr{V}_{G}(M)$.

Conjecture 10.7.1. Let $M$ be a $k G$-module. If $\mathfrak{p}$ is a homogeneous prime ideal in $H^{*}(G, k)$, we define $k(\mathfrak{p})$ to be the homogeneous field of fractions of 
$H^{*}(G, k)_{\mathfrak{p}}$. Then the nonmaximal homogeneous primes $\mathfrak{p}$ for which ${ }^{11}$

$$
\operatorname{Ext}_{H^{*}(G, k)_{\mathfrak{p}}}^{* *}\left(k(\mathfrak{p}), \widehat{\operatorname{Ext}}_{k G}^{*}(S, M)_{\mathfrak{p}}\right) \neq 0
$$

for some simple $k G$-module $S$ are exactly the primes corresponding to the varieties in $\mathscr{V}_{G}(M)$.

The point of this conjecture is that it provides a method for characterizing $\mathscr{V}_{G}(M)$ just in terms of $\operatorname{Ext}_{k G}^{*}(S, M)$, without having to tensor $M$ with the rather mysterious modules $\kappa_{V}$.

\section{Duality Theorems}

In this section, we describe various spectral sequences which can be interpreted as duality theorems for group cohomology. It is these theorems which demonstrate that most finitely generated graded commutative algebras are not candidates for group cohomology. The original version of the spectral sequence for finite groups appeared in [Benson and Carlson 1994b], and used multiple complexes and related finite Poincaré duality complexes of projective $k G$-modules. One consequence of the existence of this spectral sequence is that if $H^{*}(G, k)$ is Cohen-Macaulay then it is Gorenstein, with $a$-invariant zero (2.5). Even if $H^{*}(G, k)$ is not Cohen-Macaulay, the spectral sequence gives severe restrictions on the possibilities for the ring structure.

Greenlees [1995] discovered a way of using the same techniques to construct a cleaner spectral sequence of the form

$$
H_{\mathfrak{m}}^{s, t} H^{*}(G, k) \Longrightarrow H_{-s-t}(G, k)
$$

giving essentially equivalent information. We present here an alternative construction [Benson 2001] of Greenlees' spectral sequence using Rickard's idempotent modules.

(11.1) Choose a homogeneous set of parameters $\zeta_{1}, \ldots, \zeta_{r}$ for $H^{*}(G, k)$. For each $\zeta_{i}$, we truncate the triangle (9.2.1) to give a cochain complex of the form

$$
\cdots \rightarrow 0 \rightarrow k \rightarrow F_{\zeta_{i}} \rightarrow 0 \rightarrow \cdots
$$

where $k$ is in degree zero and $F_{\zeta_{i}}$ is in degree one, and the remaining terms are zero. The cohomology of this complex is $\Omega^{-1} E_{\zeta_{i}}$ concentrated in degree one. Tensoring these complexes together gives a complex $\Lambda^{*}$ of the form

$$
0 \rightarrow k \rightarrow \bigoplus_{1 \leq i \leq r} F_{\zeta_{i}} \rightarrow \cdots \rightarrow \bigotimes_{1 \leq i \leq r} F_{\zeta_{i}} \rightarrow 0
$$

\footnotetext{
${ }^{11}$ In commutative algebra, the ranks over $k(\mathfrak{p})$ of these Ext groups are called the Bass numbers.
} 
which is exact except in degree $r$, where its cohomology is

$$
\Omega^{-1} E_{\zeta_{1}} \otimes \cdots \otimes \Omega^{-1} E_{\zeta_{r}}
$$

Now $\mathscr{V}_{G}\left(\Omega^{-1} E_{\zeta_{i}}\right)$ is the set of subvarieties of the hypersurface determined by $\zeta_{i}$. So using (9.2.5), the variety of this tensor product is the intersection of these sets, which is empty. It follows using (9.2.3) that this tensor product is a projective $k G$-module.

Now let $\hat{P}_{*}$ be a Tate resolution of $k$ as a $k G$-module, and consider the double complex $\hat{E}_{0}^{* *}=\operatorname{Hom}_{k G}\left(\hat{P}_{*}, \Lambda^{*}\right)$. This double complex gives rise to two spectral sequences. If we take cohomology with respect to the differential coming from $\Lambda^{*}$ first, the $E_{1}$ page is $\operatorname{Hom}_{k G}\left(\hat{P}_{*}, H^{*}(\Lambda)\right)$. Since $H^{*}(\Lambda)$ is projective, the $E_{2}$ page is zero, and so the cohomology of the total complex Tot $\hat{E}_{0}^{* *}$ is zero.

On the other hand, if we first take cohomology with respect to the differential coming from $\hat{P}_{*}$, we obtain a spectral sequence whose $E_{1}$ page is $\hat{E}_{1}^{s, t}=$ $\hat{H}^{t}\left(G, \Lambda^{s}\right)$. Now each $\Lambda^{s}$ is a direct sum of modules of the form $F_{\zeta}$, where $\zeta$ is the product of a subset of size $s$ of $\zeta_{1}, \ldots, \zeta_{r}$. The cohomology of $F_{\zeta}$ is $\hat{H}^{*}(G, k)_{\zeta} \cong H^{*}(G, k)_{\zeta}$, and the maps are exactly the maps in the stable Koszul complex $^{12}$ for $\hat{H}^{*}(G, k)$ over $\zeta_{1}, \ldots \zeta_{r}$,

$$
\hat{E}_{1}^{* *}=C^{*}\left(\hat{H}^{*}(G, k) ; \zeta_{1}, \ldots, \zeta_{r}\right) .
$$

The stable Koszul complex computes local cohomology with respect to the maximal ideal $\mathfrak{m}$, and so we have a spectral sequence

$$
\hat{E}_{2}^{s, t}=H_{\mathfrak{m}}^{s, t} \hat{H}^{*}(G, k) \Longrightarrow 0
$$

(11.2) The spectral sequence (11.1.2) is almost, but not quite, the Greenlees spectral sequence, so we modify it as follows. Consider the subcomplex $E_{0}^{* *}$ consisting of all the terms in $\hat{E}_{0}^{* *}$ except the ones of the form $\hat{E}_{0}^{0, t}$ with $t<0$. Then we have a short exact sequence of complexes

$$
0 \rightarrow \operatorname{Tot} E_{0}^{* *} \rightarrow \operatorname{Tot} \hat{E}_{0}^{* *} \rightarrow \operatorname{Hom}_{k G}\left(\hat{P}_{*}^{-}, k\right) \rightarrow 0
$$

Since Tot $\hat{E}_{0}^{* *}$ is exact, the long exact sequence in cohomology and Tate duality give

$$
H^{n} \operatorname{Tot} E_{0}^{* *} \cong H^{n+1}\left(\operatorname{Hom}_{k G}\left(\hat{P}_{*}^{-}, k\right)\right) \cong H_{-n}(G, k) .
$$

So the spectral sequence of the double complex $E_{0}^{* *}$ has

$$
\begin{aligned}
& E_{1}^{* *}=C^{*}\left(H^{*}(G, k) ; \zeta_{1}, \ldots, \zeta_{r}\right) \\
& E_{2}^{s, t}=H_{\mathfrak{m}}^{s, t} H^{*}(G, k) \Longrightarrow H_{-s-t}(G, k) .
\end{aligned}
$$

\footnotetext{
${ }^{12}$ See for example [Bruns and Herzog 1993, § 3.5]. Sometimes the stable Koszul complex is called the Čech complex, but strictly speaking the latter name should be reserved for the complex where the degree zero term is deleted and the degrees of the remaining terms are decreased by one. This is the complex used to derive the spectral sequence (11.3.1).
} 
This is a spectral sequence of $H^{*}(G, k)$-modules; that is, the differentials

$$
d_{n}: E_{n}^{s, t} \rightarrow E_{n}^{s+n, t-n+1}
$$

are $H^{*}(G, k)$-linear. It converges because there are only a finite number of nonzero columns. This is because $E_{2}^{s, t}=0$ unless $s$ lies between the depth and the Krull dimension of $H^{*}(G, k)$. The spectral sequence (11.2.1) is isomorphic to the one constructed by Greenlees [1995], although the construction given there is slightly different. Note also that $H_{*}(G, k)$ is just the injective module $I_{\mathfrak{m}}$, so we can write this as

$$
H_{\mathfrak{m}}^{s, t} H^{*}(G, k) \Longrightarrow I_{\mathfrak{m}}
$$

The local cohomology can only be nonzero for $s$ at least the depth and at most the Krull dimension, so this spectral sequence often has only a few nonvanishing columns.

(11.3) Another variation on the construction (11.2) is as follows. Instead of eliminating just the negative part of the $s=0$ line of the $E_{0}$ page, we eliminate the whole of the $s=0$ line. Then after reindexing, we obtain a spectral sequence whose $E_{2}$ page is the Cech cohomology of the cohomology ring, and converging to Tate cohomology,

$$
\check{H}_{\mathfrak{m}}^{s, t} H^{*}(G, k) \Longrightarrow \hat{H}^{s+t}(G, k) .
$$

This is the spectral sequence described in [Greenlees 1995, Theorem 4.1].

(11.4) As an example of an application of the spectral sequence (11.2.1), consider the case where $H^{*}(G, k)$ is Cohen-Macaulay (2.5). In this case, the local cohomology $H_{\mathfrak{m}}^{s, *} H^{*}(G, k)$ is zero unless $s=r$, and the graded dual of $H_{\mathfrak{m}}^{r, *} H^{*}(G, k)$ is the canonical module $\Omega_{H^{*}(G, k)}$. So the $E_{2}$ page is only nonzero on the column $s=r$, and there is no room for differentials. It follows that the spectral sequence converges to $\left(\Omega_{H^{*}(G, k)}[r]\right)^{*}$, and so $\Omega_{H^{*}(G, k)}[r]$ is isomorphic to the graded dual of $H_{*}(G, k)$, which in turn is isomorphic to $H^{*}(G, k)$. We deduce that $\Omega_{H^{*}(G, k)} \cong H^{*}(G, k)[-r]$. It follows that $H^{*}(G, k)$ is Gorenstein with $a$-invariant zero. This gives the following theorem, which was first proved in [Benson and Carlson 1994b], using the original version of the spectral sequence.

THEOREM 11.4.1. Let $G$ be a finite group and $k$ be a field of characteristic $p$. If $H^{*}(G, k)$ is Cohen-Macaulay, then it is Gorenstein with a-invariant zero.

This theorem may be interpreted in terms of Poincaré series as follows. If we set $p_{G}(t)=\sum_{i=0}^{\infty} t^{i} \operatorname{dim} H^{i}(G, k)$ then the finite generation theorem says that $p_{G}(t)$ is a rational function of $t$ whose poles are at roots of unity. If $H^{*}(G, k)$ is Cohen-Macaulay, the theorem above implies that this rational function satisfies the functional equation $p_{G}(1 / t)=(-t)^{r} p_{G}(t)$. For this and related functional equations, see [Benson and Carlson 1994a]. 
(11.5) Another interpretation of Theorem 11.4.1 is as follows. If $\zeta_{1}, \ldots, \zeta_{r}$ is a homogeneous system of parameters for $H^{*}(G, k)$ with $\left|\zeta_{i}\right|=n_{i}$ then the quotient ring $H^{*}(G, k) /\left(\zeta_{1}, \ldots, \zeta_{r}\right)$ satisfies Poincaré duality with dualizing degree $a=$ $\sum_{i=1}^{r}\left(n_{i}-1\right)$. See the description of the quaternion group of order eight (2.4) for an explicit example of this phenomenon. Whether or not $H^{*}(G, k)$ is CohenMacaulay, it is shown in [Benson and Carlson 1994b] that there is always a nonzero element of the quotient $H^{*}(G, k) /\left(\zeta_{1}, \ldots, \zeta_{r}\right)$ in the dualizing degree $a$. In particular, we get the following corollary from that paper:

COROLlary 11.5.1. Let $G$ be a finite group and $k$ be a field of characteristic $p$. If $H^{*}(G, k)$ is a polynomial ring, then the generators are in degree one. This forces $p$ to be 2 , and $G / O_{2^{\prime}}(G)$ to be an elementary abelian 2-group.

Here, $O_{p^{\prime}}(G)$ denotes the largest normal subgroup of $G$ of order not divisible by $p$. If $k$ is a field of characteristic $p$, then the inflation map (3.2) from $H^{*}\left(G / O_{p^{\prime}}(G), k\right)$ to $H^{*}(G, k)$ is an isomorphism, so we would expect to get information only about the structure of $G / O_{p^{\prime}}(G)$ from information about the cohomology of $G$. If $p=2$ and $G \cong(\mathbb{Z} / 2)^{r}$ is elementary abelian then by (2.2.2), $H^{*}(G, k)=k\left[x_{1}, \ldots, x_{r}\right]$ is a polynomial ring on $r$ generators of degree one. In this case, $H_{\mathfrak{m}}^{s, t} H^{*}(G, k)$ vanishes except when $s=r$, and ${ }^{13}$

$$
H_{\mathfrak{m}}^{r, *} H^{*}(G, k)=k\left[x_{1}^{-1}, \ldots, x_{r}^{-1}\right],
$$

where the right hand side is graded in such a way that the identity element is in $H_{\mathfrak{m}}^{r,-r} H^{*}(G, k)$.

There are no differentials, and it is easy to see how the spectral sequence converges to the dual of the cohomology ring.

On the other hand, if $G \cong(\mathbb{Z} / p)^{r}$ with $p$ odd, then $H^{*}(G, k)=\Lambda\left(x_{1}, \ldots, x_{r}\right) \otimes$ $k\left[y_{1}, \ldots, y_{r}\right]$ is a tensor product of an exterior algebra on $r$ generators of degree one with a polynomial algebra on $r$ generators in degree two. Taking $y_{1}, \ldots, y_{r}$ as a homogeneous sequence of parameters, the exterior algebra $\Lambda\left(x_{1}, \ldots, x_{r}\right)$ is the finite Poincaré duality piece referred to in the discussion following Theorem 11.4.1. The local cohomology is again concentrated in degree $r$, and $H_{\mathfrak{m}}^{r}$ consists of $2^{r}$ copies of $k\left[y_{1}^{-1}, \ldots, y_{r}^{-1}\right]$ with generators in $H_{\mathfrak{m}}^{r,-r-i}, i=0, \ldots, r$ dual to a basis for the exterior algebra.

(11.6) As another Cohen-Macaulay example, if $G=D_{2^{n}}$ is dihedral of order $2^{n}$ then $H^{*}\left(G, \mathbb{F}_{2}\right)=\mathbb{F}_{2}[x, y, z] /(x y)$ where $x$ and $y$ have degree one and $z$ has degree two. We can take $x+y$ and $z$ as a homogeneous system of parameters, and the quotient is $H^{*}\left(G, \mathbb{F}_{2}\right) /(x+y, z)=\mathbb{F}_{2}[x] /\left(x^{2}\right)$, which satisfies Poincaré duality

\footnotetext{
${ }^{13}$ The notation $k\left[x_{1}^{-1}, \ldots, x_{r}^{-1}\right]$ is just a shorthand notation for the graded dual of the polynomial ring in $x_{1}, \ldots, x_{r}$. Beware that the notation does not transform correctly with respect to linear transformations of $x_{1}, \ldots, x_{r}$ because it depends on the choice of system of parameters for the stable Koszul complex. The action on the inverse generators should be transposed from what the notation suggests. However, the notion is called "Macaulay's inverse system," and is standard in commutative algebra.
} 
with dualizing degree one. The local cohomology is concentrated in degree two, and consists of two copies of $\mathbb{F}_{2}\left[(x+y)^{-1}, z^{-1}\right]$ with generators in $H_{\mathfrak{m}}^{2,-2}$ and $H_{\mathfrak{m}}^{2,-3}$ dual to 1 and $x$.

(11.7) If $G=S D_{2^{n}}$ is semidihedral of order $2^{n}$ (2.8.1) and $k$ is a field of characteristic two then $H^{*}(G, k)=k[x, y, z, w] /\left(x y, y^{3}, y z, z^{2}+w x^{2}\right)$ where $x$ and $y$ have degree one, $z$ has degree three, and $w$ has degree four. The depth of this ring is one, and the Krull dimension is two, so there is local cohomology in degrees one and two; $H_{\mathfrak{m}}^{1}$ consists of two copies of $\mathbb{F}_{2}\left[w^{-1}\right]$ with generators in $H_{\mathfrak{m}}^{1,-2}$ and $H_{\mathfrak{m}}^{1,-3}$ which play the role of duals for $y$ and $y^{2}$, while $H_{\mathfrak{m}}^{2}$ consists of two copies of $\mathbb{F}_{2}\left[w^{-1}, x^{-1}\right]$ with generators in $H_{\mathfrak{m}}^{2,-2}$ and $H_{\mathfrak{m}}^{2,-5}$ dual to 1 and $z$. This example is described in more detail in $\S 2$.

(11.8) The cohomology of the 2-groups of order at most 32 has been calculated by Rusin [1989]. A particularly interesting example is the group

$$
\begin{aligned}
\Gamma_{7} a_{2}=\langle a, b, c| a^{4} b= & b a^{4}, a^{4} c=c a^{4}, b c=c b, \\
& \left.a^{8}=b^{2}=c^{2}=1, a b a^{-1}=b c, a c a^{-1}=a^{4} c\right\rangle,
\end{aligned}
$$

of order 32, whose cohomology has Krull dimension three and depth one. This is the smallest example where the Greenlees spectral sequence has a nonzero differential. The cohomology ring $H^{*}\left(\Gamma_{7} a_{2}, \mathbb{F}_{2}\right)$ is generated by elements $z, y, x$, $w, v, u, t$ and $s$ of degrees $1,1,2,2,3,3,4,4$, respectively, where the ideal of relations is generated by the elements

$$
\begin{aligned}
& z y, y^{2}, y x, y w, y v, y u, y t, x w+z u, z^{2} w+w^{2}, w v+z t, z x w+w u \\
& z^{2} t+w t, x^{3}+z x v+z^{2} s+v^{2}, v u+x t, x^{2} u+z x t+z w s+v t \\
& x^{3} w+z^{2} x t+z^{2} w s+t^{2}, z x t+u t, x^{2} w+u^{2}
\end{aligned}
$$

As a module over the polynomial subring $\mathbb{F}_{2}[z, x, s]$, the cohomology is generated by $1, y, w, v, u$ and $t$, subject to the relations $z y=0, x y=0$ and $z u=x w$. The local cohomology is nonvanishing in degrees 1,2 and 3 ; both $H_{\mathfrak{m}}^{1}$ and $H_{\mathfrak{m}}^{2}$ consist of a copy of $\mathbb{F}_{2}\left[s^{-1}\right]$ generated in degree -3 , while $H_{\mathfrak{m}}^{3}$ consists of four copies of $\mathbb{F}_{2}\left[z^{-1}, x^{-1}, s^{-1}\right]$ generated in degrees $-3,-4,-6$ and -7 . The nonzero differential $d_{2}$ takes the generator in $H_{\mathfrak{m}}^{1,-3}$ to the generator in $H_{\mathfrak{m}}^{3,-4}$, wiping out $H_{\mathfrak{m}}^{1}$ and having a cokernel on this part of $H_{\mathfrak{m}}^{3}$ which plays the role of the dual of the summand generated by $w$ and $u$. The remaining generators in $H_{\mathfrak{m}}^{3,-3}, H_{\mathfrak{m}}^{3,-6}$ and $H_{\mathfrak{m}}^{3,-7}$ are dual to $1, v$ and $t$, while the generator of $H_{\mathfrak{m}}^{2,-3}$ is dual to $y$.

\section{More Duality Theorems}

(12.1) There is a version of the spectral sequence (11.2.1) for compact Lie groups [Benson and Greenlees 1997a]. This involves a dimension shift, equal to the dimension $d$ of $G$ as a manifold. There is also an orientation issue. Namely, 
the adjoint representation of $G$ gives a homomorphism $G \rightarrow \mathrm{O}(d)$, whose image does not necessarily lie in $\mathrm{SO}(d)$. Composing with the determinant representation of $\mathrm{O}(d)$, which takes values \pm 1 , gives a one dimensional representation $\varepsilon: \pi_{1}(B G) \cong \pi_{0}(G) \rightarrow k^{\times}$. The spectral sequence then takes the form

$$
H_{\mathfrak{m}}^{s, t} H^{*}(B G ; k) \Longrightarrow H_{-d-s-t}(B G ; \varepsilon) .
$$

Dwyer, Greenlees and Iyengar [Dwyer et al. 2002] give another proof for compact Lie groups and also a version for $p$-compact groups.

For example, if $H^{*}(B G ; k)$ is a polynomial ring on generators $\zeta_{1}, \ldots, \zeta_{r}$ with $\left|\zeta_{i}\right|=n_{i}$, and $\varepsilon=k$, then we have

$$
d=\sum_{i=1}^{r}\left(n_{i}-1\right)
$$

If $G=\mathrm{U}(n)$, the compact unitary group of $n \times n$ matrices, and $k$ is any commutative coefficient ring, then $H^{*}(B \mathrm{U} ; k)=k\left[c_{1}, \ldots, c_{n}\right]$ is a polynomial ring on Chern classes $c_{i}$ of degree $2 i$ (3.3.1). In accordance with equation (12.1.2), we have $\operatorname{dim} \mathrm{U}(n)=n^{2}=\sum_{i=1}^{n}(2 i-1)$.

On the other hand, if $G=\mathrm{O}(2 n)$, the compact orthogonal group of real $2 n \times 2 n$ matrices preserving a positive definite inner product, and $k$ is a field of characteristic not equal to two, then $H^{*}(B \mathrm{O}(2 n) ; k)=k\left[p_{1}, \ldots, p_{n}\right]$ is a polynomial ring on Pontrjagin classes $p_{i}$ of degree $4 i$ (3.3.2). Since $\operatorname{dim} \mathrm{O}(2 n)=2 n^{2}-n$ and $\sum_{i=1}^{n}(4 i-1)=2 n^{2}+n$, we see that the two sides of equation (12.1.2) differ by $2 n$. This is because the orientation representation $\varepsilon$ is nontrivial, and

$$
H^{*}(B \mathrm{O}(2 n) ; \varepsilon)=k\left[p_{1}, \ldots, p_{n}\right] \cdot e
$$

where $e \in H^{2 n}(B \mathrm{SO}(2 n) ; k) \cong H^{2 n}(B \mathrm{O}(2 n) ; k \oplus \varepsilon)$ is the Euler class, satisfying $e^{2}=p_{n}$ (3.3.3). The degree of the Euler class exactly accounts for the discrepancy in equation (12.1.2).

(12.2) Another version of the spectral sequence has been developed for virtual duality groups [Benson and Greenlees 1997b]. The latter is a class of groups which includes arithmetic groups [Borel and Serre 1973], mapping class groups of orientable surfaces [Harer 1986] and automorphism groups of free groups of finite rank [Bestvina and Feighn 2000]. A discrete group $G$ is said to be a duality group of dimension $d$ over $k$ (see [Bieri 1976]) if there is a dualizing module. This is defined to be a $k G$-module $I$ such that there are isomorphisms $H^{i}(G, M) \cong H_{d-i}\left(G, I \otimes_{k} M\right)$ for all $k G$-modules $M$. It turns out that such isomorphisms may be taken to be functorial in $M$ if they exist at all, and in that case, $I \cong H^{d}(G, k G)$. A Poincaré duality group is a duality group for which the dualizing module $I$ is isomorphic to the field $k$ with some $G$-action, and it is orientable if the action is trivial. A virtual duality group of dimension $d$ is a group $G$ with a normal subgroup $N$ of finite index which is a duality group of dimension $d$. Since the Eckmann-Shapiro lemma says that $H^{*}(G, k G) \cong H^{*}(N, k N)$, the 
dualizing module $I$ does not depend on which normal subgroup is used in the definition. The spectral sequence for a virtual duality group takes the form

$$
H_{\mathfrak{m}}^{s, t} H^{*}(G, M) \Longrightarrow H_{d-s-t}(G, I \otimes M)
$$

Notice that the sign of the degree shift in this case is in the opposite direction to the case of a compact Lie group. So a virtual Poincaré duality group of dimension $d$ behaves very much like a compact Lie group of dimension $-d$.

(12.3) In [Greenlees 2002, § 8.4], there is a brief discussion of the corresponding version for continuous cohomology of $p$-adic Lie groups, which are a particular kind of profinite groups. These include matrix groups over the $p$-adic integers such as $\operatorname{SL}\left(n, \mathbb{Z}_{p}^{\wedge}\right)$. The discussion for $p$-adic Lie groups translates into continuous cohomology the story for virtual duality groups, with the same shift in dimension. The way this works is as follows. By [Lazard 1965, Chapter V, 2.2.7.1 and 2.5.7.1], if $G$ is a $p$-adic Lie group then $G$ has a normal open subgroup $H$ for which $H_{c}^{*}\left(H, \mathbb{F}_{p}\right)$ is the exterior algebra on $H_{c}^{1}\left(H, \mathbb{F}_{p}\right)$, so that $H$ is a Poincaré duality group. Furthermore, $H_{c}^{1}\left(H, \mathbb{F}_{p}\right)$ is a finite dimensional $\mathbb{F}_{p}$-vector space whose dimension is equal to the dimension $d$ of $G$ as a $p$-adic manifold. So the dualizing module $\varepsilon$ is the $\mathbb{F}_{p} G$-module $H_{c}^{d}\left(H, \mathbb{F}_{p}\right)$, which is the same as the determinant of the adjoint representation of $G$ on its Lie algebra. The spectral sequence then takes the form

$$
H_{\mathfrak{m}}^{s, t} H_{c}^{*}\left(G, \mathbb{F}_{p}\right) \Longrightarrow H_{d-s-t}^{c}(G, \varepsilon) .
$$

(12.4) There are also versions of the spectral sequence for other cohomology theories. For example, [Bruner and Greenlees 2003] investigates the spectral sequence

$$
H_{I}^{*, *} k u^{*}(B G) \Longrightarrow k u_{*}(B G)
$$

where $k u$ denotes connective complex $K$-theory, $I$ is the kernel of the augmentation map $k u^{*}(B G) \rightarrow k u^{*}$, and $G$ is a finite group.

(12.5) The papers [Dwyer et al. 2002; Greenlees 2002] also explain a more general context for some of these spectral sequences. They explain the sense in which the cochains on $B G$ and related objects are examples of Gorenstein differential graded algebras. Their notions are expressed in the language of $E_{\infty}$ ring spectra, or commutative $S$-algebras, see [Elmendorf et al. 1997].

\section{Dual Localization}

(13.1) Greenlees and Lyubeznik [2000] introduced a way of obtaining information at nonmaximal prime ideals out of the Greenlees spectral sequence. Roughly speaking, one would like to localize the spectral sequence. Attempting to do this directly turns out to be a bad move. The reason is that every element of $H_{\mathfrak{m}}^{* *} H^{*}(G, k)$ and every element of $I_{\mathfrak{m}}$ is killed by some power of $\mathfrak{m}$. So the idea 
is to dualize first, then localize, and then dualize back again. The dualization process needed for this is graded Matlis duality. See [Matlis 1958] for ordinary Matlis duality, and [Bruns and Herzog 1993, $\S 3.6$ ] for the graded version. If $\mathfrak{p}$ is a homogeneous prime ideal in $H^{*}(G, k)$ and $X$ is a module over $H^{*}(G, k)_{\mathfrak{p}}$, then we write $D_{\mathfrak{p}}(X)$ for the graded Matlis dual of $X$

$$
D_{\mathfrak{p}}(X)=\operatorname{Hom}_{H^{*}(G, k)_{\mathfrak{p}}}\left(X, I_{\mathfrak{p}}\right),
$$

where $I_{\mathfrak{p}}$ is the injective hull of $H^{*}(G, k) / \mathfrak{p}$. The latter can be viewed as a module over the completion $H^{*}(G, k)_{\mathfrak{p}}^{\wedge}$, and so $D_{\mathfrak{p}}$ takes $H^{*}(G, k)_{\mathfrak{p}}$-modules to $H^{*}(G, k)_{\mathfrak{p}}^{\wedge}$-modules. It takes Artinian modules to Noetherian modules, and viceversa. Applying $D_{\mathfrak{p}}$ twice to an Artinian module returns the same module, and applying $D_{\mathfrak{p}}$ twice to a Noetherian module returns its $\mathfrak{p}$-adic completion. In this language, we can rewrite equation (10.5.3) as

$$
D_{\mathfrak{p}} \hat{H}^{*}(G, M) \cong \widehat{\operatorname{Ext}}_{k G}^{*}\left(M, T\left(I_{\mathfrak{p}}\right)\right) .
$$

Tate duality is the special case of this statement where $\mathfrak{p}=\mathfrak{m}$, because $D_{\mathfrak{m}}$ can be interpreted as taking the graded dual of a graded vector space, and $T\left(I_{\mathfrak{m}}\right)=\Omega(k)$.

Grothendieck duality [Grothendieck 1965; 1967] says that if we choose a polynomial subring $R=k\left[\zeta_{1}, \ldots, \zeta_{r}\right]$ over which $H^{*}(G, k)$ is finitely generated as a module, and $M$ is a graded $H^{*}(G, k)$-module, then the graded Matlis dual of local cohomology is Ext over $R$ in complementary degrees,

$$
D_{\mathfrak{m}} H_{\mathfrak{m}}^{s, t} M \cong \operatorname{Ext}_{R}^{r-s,-t}(M, R[-a])
$$

where $a=\sum_{i=1}^{r}\left|\zeta_{i}\right|$ and $R[-a]$ is the canonical module for $R$. So the graded Matlis dual of the Greenlees spectral sequence is

$$
\operatorname{Ext}_{R}^{r-s,-t}\left(H^{*}(G, k), R[-a]\right) \Longrightarrow H^{-s-t}(G, k) .
$$

Localizing this spectral sequence with respect to a homogeneous prime ideal $\mathfrak{p} \neq \mathfrak{m}$ of dimension $d$ gives a spectral sequence

$$
\operatorname{Ext}_{R_{\mathfrak{q}}}^{r-s,-t}\left(H^{*}(G, k)_{\mathfrak{p}}, R_{\mathfrak{q}}[-a]\right) \Longrightarrow H^{-s-t}(G, k)_{\mathfrak{p}}
$$

where $\mathfrak{q}=\mathfrak{p} \cap R$. Since $R_{\mathfrak{q}}$ has Krull dimension $r-d$ instead of $r$, applying $D_{\mathfrak{p}}$ to this spectral sequence and using Grothendieck duality again gives a spectral sequence of the form $H_{\mathfrak{p}}^{s-d, t} H^{*}(G, k)_{\mathfrak{p}} \Longrightarrow I_{\mathfrak{p}}$, or reindexing,

$$
H_{\mathfrak{p}}^{s, t} H^{*}(G, k)_{\mathfrak{p}} \Longrightarrow I_{\mathfrak{p}}[d] \text {. }
$$

This is the Greenlees-Lyubeznik dual localized form of the Greenlees spectral sequence. So for example, taking $\mathfrak{p}$ to be a minimal prime in $H^{*}(G, k)$, this spectral sequence has only one nonvanishing column, and it follows that $H^{*}(G, k)_{\mathfrak{p}}$ is Gorenstein. This gives the following theorem. 
THEOREM 13.1.3. If $G$ is a finite group and $k$ is a field then $H^{*}(G, k)$ is generically Gorenstein.

(13.2) There is another, more module theoretic method for getting a spectral sequence with the same $E_{2}$ page as (13.1.2), described in [Benson 2001]. Let $V \subseteq V_{G}$ be the closed homogeneous irreducible subvariety corresponding to $\mathfrak{p}$, and let $\mathscr{W}$ be the subset of Proj $H^{*}(G, k)$ used in the definition (9.2.2) of $\kappa_{V}$. Since the maximal elements of $\mathscr{W}$ have codimension one in $V_{G}$, the cohomology of the $F$-idempotent is just the homogeneous localization, $\hat{H}^{*}\left(G, F_{\mathscr{W}}\right)=H^{*}(G, k)_{\mathfrak{p}}$. Let $h$ be the height of $\mathfrak{p}$, namely the Krull dimension of $H^{*}(G, k)_{\mathfrak{p}}$. Then by the version of the Noether normalization theorem described in [Nagata 1962], we can choose a homogeneous set of parameters $\zeta_{1}, \ldots, \zeta_{r}$ for $H^{*}(G, k)$ so that $\zeta_{1}, \ldots, \zeta_{h}$ lie in $\mathfrak{p}$. So $\zeta_{1}, \ldots, \zeta_{h}$ is a system of parameters for $H^{*}(G, k)_{\mathfrak{p}}$. We tensor together the complexes (11.1.1) for $\zeta_{1}, \ldots, \zeta_{h}$ to obtain a complex $\Lambda^{*}\left(\zeta_{1}, \ldots, \zeta_{h}\right)$ of the form

$$
0 \rightarrow k \rightarrow \bigoplus_{i=1}^{h} F_{\zeta_{i}} \rightarrow \cdots \rightarrow \bigotimes_{i=1}^{h} F_{\zeta_{i}} \rightarrow 0
$$

and then tensor the answer with the module $F_{\mathscr{W}}$ to obtain a complex

$$
\Lambda_{\mathfrak{p}}^{*}=\Lambda^{*}\left(\zeta_{1}, \ldots, \zeta_{h}\right) \otimes F_{\mathscr{W}}
$$

whose cohomology is $\Omega^{-h} E_{V} \otimes F_{\mathscr{W}}=\Omega^{-h} \kappa_{V}$ concentrated in degree $h$. The spectral sequence of the double complex $E_{0}^{* *}(\mathfrak{p})=\operatorname{Hom}_{k G}\left(\hat{P}_{*}, \Lambda_{\mathfrak{p}}^{*}\right)$ gives

$$
E_{2}^{s, t}(\mathfrak{p})=H_{\mathfrak{p}}^{s, t} H^{*}(G, k)_{\mathfrak{p}} \Longrightarrow \hat{H}^{s+t}\left(G, \kappa_{V}\right) .
$$

Conjecture 13.2.2. The spectral sequences 13.1 .2 and 13.2 .1 are isomorphic from the $E_{2}$ page onwards.

It is proved in [Benson 2001] that Conjecture 13.2.2 implies Conjecture 10.6.1. Furthermore, Conjecture 13.2.2 clearly holds in the case where $H^{*}(G, k)$ is Cohen-Macaulay, because there is no room for nontrivial differentials or ungrading problems.

\section{Quasiregular Sequences}

In this section, we describe the theory of quasiregular sequences, first introduced in [Benson and Carlson 1994b], and describe their relationship with the local cohomology of $H^{*}(G, k)$. The material of this section is further developed in a companion paper [Benson 2004], written during the month following the MSRI workshop.

(14.1) Let $G$ be a finite group of $p$-rank $r$, and let $k$ be a field of characteristic p. A homogeneous sequence of parameters $\zeta_{1}, \ldots, \zeta_{r}$ for $H^{*}(G, k)$ with $\left|\zeta_{i}\right|=n_{i}$ is said to be filter-regular if for each $i=0, \ldots, r-1$, the map

$$
\left(H^{*}(G, k) /\left(\zeta_{1}, \ldots, \zeta_{i}\right)\right)^{j} \rightarrow\left(H^{*}(G, k) /\left(\zeta_{1}, \ldots, \zeta_{i}\right)\right)^{j+n_{i+1}}
$$


induced by multiplication by $\zeta_{i+1}$ is injective for $j$ large enough. The existence of a filter-regular sequence is guaranteed by the standard method of prime avoidance.

In [Benson and Carlson 1994b, §10], the following terminology was introduced. A sequence of parameters $\zeta_{1}, \ldots, \zeta_{r}$ is said to be quasiregular ${ }^{14}$ if the map (14.1.1) is injective for $i=0, \ldots, r-1$ whenever $j \geq n_{1}+\cdots+n_{i}$, and $H^{*}(G, k) /\left(\zeta_{1}, \ldots, \zeta_{r}\right)$ is zero in degrees at least $n_{1}+\cdots+n_{r}$. For $i=0$ this is the same as saying that $\zeta_{1}$ is a regular element, but for $i>0$ it allows some low degree kernel.

Conjecture 14.1.2. For any finite group $G$ and field $k$, there exists a quasiregular sequence in $H^{*}(G, k)$.

It is proved in [Benson and Carlson 1994b] that the conjecture is true if $r \leq 2$, and Okuyama and Sasaki [2000] have a proof for $r \leq 3$. These proofs work more generally when the depth and Krull dimension differ by at most one, respectively two. In this section, I shall try to explain the ideas behind these proofs, and the relevance of quasiregular sequences for the computation of group cohomology.

(14.2) We can reinterpret the definition of quasiregular sequence in terms of cohomology of modules as follows, and in the process give some sort of explanation of where the condition $j \geq n_{1}+\cdots+n_{i}$ comes from. We can always take our first parameter $\zeta_{1}$ to be a regular element, by Duflot's Theorem 6.2.1. Consider the short exact sequence

$$
0 \rightarrow L_{\zeta_{1}} \rightarrow \Omega^{n_{1}}(k) \rightarrow k \rightarrow 0 .
$$

The long exact sequence in cohomology gives (for $j \geq n_{1}$ ) an exact sequence

$$
\cdots \rightarrow H^{j}\left(G, L_{\zeta_{1}}\right) \rightarrow H^{j-n_{1}}(G, k) \stackrel{\zeta_{1}}{\longrightarrow} H^{j}(G, k) \rightarrow H^{j+1}\left(G, L_{\zeta_{1}}\right) \rightarrow \cdots
$$

So we have $H^{j+1}\left(G, L_{\zeta_{1}}\right) \cong\left(H^{*}(G, k) /\left(\zeta_{1}\right)\right)^{j}$ for $j \geq n_{1}$.

Working inductively, for each $i=0, \ldots, r-1$, if we tensor the short exact sequence

$$
0 \rightarrow L_{\zeta_{i+1}} \rightarrow \Omega^{n_{i+1}}(k) \rightarrow k \rightarrow 0
$$

with the module $M_{i}=L_{\zeta_{1}} \otimes \cdots \otimes L_{\zeta_{i}}$ and take the long exact sequence in cohomology, then we obtain the following.

Proposition 14.2.1. A homogeneous sequence of parameters $\zeta_{1}, \ldots, \zeta_{r}$ is quasiregular if and only if for each $i=0, \ldots, r-1$, multiplication by $\zeta_{i+1}$ is injective on $H^{j}\left(G, M_{i}\right)$ for $j \geq n_{1}+\cdots+n_{i}+i$.

${ }^{14}$ This terminology has nothing to do with the terminology of quasiregular sequences used in [Matsumura 1989]. The definition in [Benson and Carlson 1994b] omits the condition on $H^{*}(G, k) /\left(\zeta_{1}, \ldots, \zeta_{r}\right)$, but this condition turns out to be automatic, see Corollary 14.2.2. 
Corollary 14.2.2. If $\zeta_{1}, \ldots, \zeta_{r}$ is a homogeneous system of parameters and the condition for quasiregularity is satisfied for $i=0, \ldots, r-2$, then it is also satisfied for $i=r-1$, and the quotient $H^{*}(G, k) /\left(\zeta_{1}, \ldots, \zeta_{r}\right)$ is zero in degree at least $n_{1}+\cdots+n_{r}$.

Proof. $H^{j+r-1}\left(G, M_{r-1}\right)=\left(H^{*}(G, k) /\left(\zeta_{1}, \ldots, \zeta_{r-1}\right)\right)^{j}$ for $j \geq n_{1}+\cdots+n_{r-1}$. Tensoring the sequence $0 \rightarrow L_{\zeta_{r}} \rightarrow \Omega^{n_{r}} k \stackrel{\hat{\zeta}_{r}}{\longrightarrow} k \rightarrow 0$ with $M_{r-1}$, and using the fact that $M_{r-1} \otimes L_{\zeta_{r}}$ is projective, we see that $\zeta_{r}$ induces an isomorphism $\Omega^{n_{r}} M_{r-1} \cong M_{r-1}$, and hence an isomorphism on $H^{*}\left(G, M_{r-1}\right)$ in positive degrees.

(14.3) To go further, we make use of the transfer map. If we choose the parameters to be the Dickson invariants (see $\S 7$ ), then the restriction to each elementary abelian $p$-subgroup $E$ of rank $r-1$ of the sequence $\zeta_{1}, \ldots, \zeta_{r-1}$ is a homogeneous sequence of parameters in $H^{*}(E, k)$. It follows that $V_{G}\left(M_{r-1}\right)$ has trivial intersection with the image of $V_{E} \rightarrow V_{G}$ for each such $E$. Theorem 1.5 of [Benson 1994/95] (see also Corollary 4.5 of [Carlson et al. 1998]) then shows that $M_{r-2} \otimes L_{\zeta_{r-1}}=M_{r-1}$ is projective relative to ${ }^{15}$ the set $\mathscr{H}_{r}$ of centralizers $C_{G}(E)$ of elementary abelian $p$-subgroups $E$ of rank $r$. So the sum of the transfers from these subgroups gives a surjective map in cohomology. Furthermore, the restrictions of $\zeta_{1}, \ldots, \zeta_{r}$ to a subgroup in $\mathscr{H}_{r}$ form a regular sequence, by Duflot's theorem. Now examine the diagram

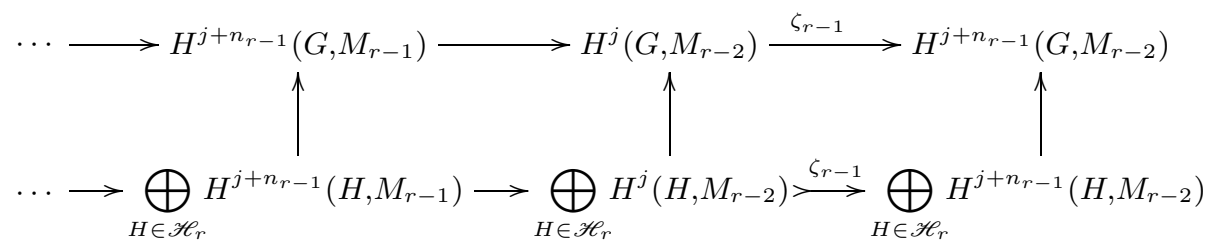

where the vertical maps are given by $\sum_{H \in \mathscr{H}_{r}} \operatorname{Tr}_{H, G}$. The map marked $\zeta_{r-1}$ on the bottom row is injective, and a diagram chase shows that the corresponding map on the top row is therefore also injective. So $\zeta_{r-1}$ is quasiregular. Finally, the argument of the previous paragraph shows that the last parameter $\zeta_{r}$ is also quasiregular. This completes the argument of Okuyama and Sasaki, proving Conjecture 14.1.2 in the case where the depth and the Krull dimension differ by at most two.

It looks as though the argument above ought to admit a modification which makes it work inductively and prove the conjecture, but so far nobody has succeeded in doing this.

Carlson [1999; 2001] has developed some conjectures related to Conjecture 14.1.2, which allow a machine computation of group cohomology by computing

\footnotetext{
${ }^{15} \mathrm{~A}$ module $M$ is said to be projective relative to a set of subgroups $\mathscr{H}$ of $G$ if it is a direct summand of a direct sum of modules induced from elements of $\mathscr{H}$. This is equivalent to the statement that the sum of the transfer maps $\operatorname{Tr}_{H, G}: \operatorname{End}_{k H}(M) \rightarrow \operatorname{End}_{k G}(M)$ from the subgroups $H \in \mathscr{H}$ is surjective. For further details, see [Benson 1991a, §3.6].
} 
just a finite part at the beginning of a projective resolution. The usefulness of the conjectures depends on the fact that during the course of the calculation for a particular group, it is proved that the cohomology ring really does satisfy the conjectures, so there is no uncertainty about the answer. Condition G of [Carlson 2001 is related to the existence of a quasiregular sequence, while Condition $\mathrm{R}$ of that paper is a weak form of Conjecture 7.2.1. If the existence of a quasiregular sequence could be verified a priori, then the computational method could be guaranteed to work. This is explained in Theorem 14.5.2 below.

The cohomology of the groups of order 64 can be found in [Carlson $\geq 2004$ ]. In the course of the computations, Conditions G and R of [Carlson 2001] were verified for these groups.

(14.4) The existence of a quasiregular sequence in group cohomology can be reformulated in terms of local cohomology as follows. If

$$
H=\bigoplus_{i \geq 0} H^{i}=k \oplus \mathfrak{m}
$$

is a graded commutative ring with $H^{0}=k$ a field and $\mathfrak{m}=\bigoplus_{i>0} H^{i}$, and $M$ is a graded $H$-module, we set

$$
a_{\mathfrak{m}}^{i}(M)=\max \left\{n \in \mathbb{Z} \mid H_{\mathfrak{m}}^{i, n}(M) \neq 0\right\}
$$

(or $a_{\mathfrak{m}}^{i}(M)=-\infty$ if $H_{\mathfrak{m}}^{i}(M)=0$ ).

The following is proved in Corollary 3.7 of [Benson 2004].

THEOREM 14.4.1. If $G$ is a finite group and $k$ is a field, then the following are equivalent.

(i) There is a quasiregular sequence in $H^{*}(G, k)$,

(ii) Every filter-regular sequence of parameters in $H^{*}(G, k)$ is quasiregular,

(iii) The Dickson invariants (see $\S 7)$ in $H^{*}(G, k)$ are quasiregular,

(iv) For all $i \geq 0$ we have $a_{\mathfrak{m}}^{i}\left(H^{*}(G, k)\right)<0$.

(14.5) It is shown in [Benson 2004, $\S 5$ ] that we can interpret the invariants $a_{\mathfrak{m}}^{i}$ in terms of resolutions. If $R=k\left[\zeta_{1}, \ldots, \zeta_{r}\right]$ is a polynomial subring over which $H$ is finitely generated as a module, and $M$ is a graded $H$-module, let

$$
0 \rightarrow F_{r} \rightarrow \cdots \rightarrow F_{0} \rightarrow M \rightarrow 0
$$

be a minimal resolution of $M$ over $R$. We define $\beta_{j}^{R}(M)$ to be the largest degree of a generator of $F_{j}$ as an $R$-module (or $\beta_{j}^{R}(M)=-\infty$ if $F_{j}=0$ ). Then we have

$$
\max _{j \geq 0}\left\{a_{\mathfrak{m}}^{j}(M)\right\}=\max _{j \geq 0}\left\{\beta_{j}^{R}(M)-\sum_{i=1}^{r}\left|\zeta_{i}\right|\right\} .
$$

This equation, together with Theorem 14.4.1, explains the relevance of the existence of quasiregular sequences to finding bounds for the degrees of generators 
and relations for the group cohomology. Better ways of bounding the degrees of the relations can be found in [Carlson 2001] and in [Benson 2004].

TheOREM 14.5.2. Let $G$ be a finite group and $k$ be a field. If $\zeta_{1}, \ldots, \zeta_{r}$ is a quasiregular sequence in $H^{*}(G, k)$, then all the generators for $H^{*}(G, k)$ have degree at most $\sum_{i=1}^{r}\left|\zeta_{i}\right|$, and the relations have degree at most $2\left(\sum_{i=1}^{r}\left|\zeta_{i}\right|\right)-2$.

Proof. Set $R=k\left[\zeta_{1}, \ldots, \zeta_{r}\right]$ as above. By Theorem 14.4.1, the existence of a quasiregular sequence implies that $a_{\mathfrak{m}}^{j}\left(H^{*}(G, k)\right)<0$ for all $j \geq 0$. So by equation (14.5.1), we have

$$
\beta_{j}^{R}\left(H^{*}(G, k)\right)<\sum_{i=1}^{r}\left|\zeta_{i}\right|
$$

for all $j \geq 0$. The numbers $\beta_{0}^{R}$ and $\beta_{1}^{R}$ are the largest degrees for generators and relations respectively of $H^{*}(G, k)$ as an $R$-module. The ring generators have degree at most $\max \left(\beta_{0}^{R},\left|\zeta_{1}\right|, \ldots,\left|\zeta_{r}\right|\right)$. For the ring relations, we need the $R$-module relations together with relations saying how the products of pairs of $R$-module generators can be written as $R$-linear combinations of generators.

(14.6) The Castelnuovo-Mumford regularity of a graded $H$-module $M$ (see [Eisenbud 1995, § 20.5], for example) is defined as

$$
\operatorname{Reg} M=\max _{j \geq 0}\left\{a_{\mathfrak{m}}^{j}(M)+j\right\}=\max _{j \geq 0}\left\{\beta_{j}^{R}(M)-j-\sum_{i=1}^{r}\left(\left|\zeta_{i}\right|-1\right)\right\} .
$$

The second equality here is proved in [Benson 2004]. Usually the summation term does not appear, because much of the literature on the subject assumes that the graded ring $H$ is generated over $H^{0}$ by elements of degree one; in this context the above equality was proved in [Eisenbud and Goto 1984].

The "last survivor" described in [Benson and Carlson 1994b, Theorem 1.3] and reinterpreted in terms of local cohomology in [Benson 2001, Theorem 4.1] says that for a finite group $G$ over a field $k$ we have $H_{\mathfrak{m}}^{r,-r} H^{*}(G, k) \neq 0$, so that $\operatorname{Reg} H^{*}(G, k) \geq 0$. One might strengthen Conjecture 14.1.2 to the following statement, which has been checked for the 2-groups of order at most 64 using Carlson's calculations $[\geq 2004]$.

Conjecture 14.6.1. If $G$ is a finite group and $k$ is a field then $\operatorname{Reg} H^{*}(G, k)=0$.

This conjecture is equivalent to a strengthening of the bound given in the definition of a quasiregular sequence to $j>n_{1}+\cdots+n_{i}-i$. Pushing the argument given in the proof of Proposition 14.2.1 to its limits, and using some subtle information about $\hat{H}^{-1}(G, k)$, one can translate this into a strengthening of the module theoretic bounds given in that proposition to $j>n_{1}+\cdots+n_{i}$. For the details, see [Benson 2004].

EXAMPLE 14.6.2. Let $G$ be the Sylow 2-subgroup of PSL $\left(3, \mathbb{F}_{4}\right)$, of order 64 (this is group number 183 in the Appendix). Then $H^{*}\left(G, \mathbb{F}_{2}\right)$ has Krull dimension four 
and depth two, with $a_{\mathfrak{m}}^{2}=-3, a_{\mathfrak{m}}^{3}=-5$ and $a_{\mathfrak{m}}^{4}=-4$. So the regularity is zero. This is the only example of a 2 -group of order at most 64 where $H_{\mathfrak{m}}^{r,-r} H^{*}\left(G, \mathbb{F}_{2}\right)$ has dimension bigger than one; in this example it has dimension two.

(14.7) Conjecture 14.6.1 can be interpreted in terms of the Greenlees spectral sequence (11.2.1). It says that the $E_{2}$ page vanishes above the line $s+t=0$. Of course, this part of the $E_{2}$ page dies by the time the $E_{\infty}$ page is reached, in order for the spectral sequence to be able to converge to a negatively graded target. In the above example, the extra dimension in $H_{\mathfrak{m}}^{4,-4}$ has to be hit in the spectral sequence by $H_{\mathfrak{m}}^{2,-3}$.

The conjecture can be generalized to compact Lie groups, virtual duality groups and $p$-adic Lie groups as follows.

Conjecture 14.7.1. If $G$ is a compact Lie group of dimension $d$ and $k$ is a field then $\operatorname{Reg} H^{*}(B G ; \varepsilon)=-d$. Here, $\varepsilon$ is the orientation representation of (12.1.1).

Conjecture 14.7.2. If $G$ is an orientable virtual Poincaré duality group of dimension $d$ over a field $k$ then $\operatorname{Reg} H^{*}(G, k)=d$.

Conjecture 14.7.3. If $G$ is a $p$-adic Lie group of dimension $d$ then over $\mathbb{F}_{p}$ we have $\operatorname{Reg} H^{*}(G, \varepsilon)=d$. Here, $\varepsilon$ is the the orientation representation of (12.3.1).

As a nontrivial example, for the compact simply connected Lie group $E_{6}$ of dimension 78, the calculations of Kono and Mimura [1975] (see also [Benson and Greenlees 1997a]) imply that $H^{*}\left(B E_{6} ; \mathbb{F}_{2}\right)$ has Krull dimension six and depth five, with $a_{\mathfrak{m}}^{5}=-90$ and $a_{\mathfrak{m}}^{6}=-84$, so that $\operatorname{Reg} H^{*}\left(B E_{6} ; \mathbb{F}_{2}\right)=-78$.

\section{Appendix: Two-Groups of Order 64 and Their $\bmod 2$ Cohomology}

The table on the next page lists the Krull dimension of $H^{*}\left(G, \mathbb{F}_{2}\right)$, the depth of $H^{*}\left(G, \mathbb{F}_{2}\right)$, and the rank of the center of $G$ (see Duflot's Theorem 6.2.1), for each of the 2-groups $G$ of order 64 . The numbering of the groups follows that of Hall and Senior [1964], who classified these groups. Underlined entries have

$$
\text { Krull dimension }- \text { depth }=2 \text {; }
$$

otherwise the difference is 1 or 0 .

A separate table on page 45 gives the invariants $a_{\mathfrak{m}}^{i}\left(H^{*}\left(G, \mathbb{F}_{2}\right)\right)$ defined in (14.4), for the entries where the difference is 2, with the rows of the table arranged in decreasing order of Krull dimension. Note that Duflot's Theorem 6.2.1 implies that $a_{\mathfrak{m}}^{0}$ is always zero, so the tables begin with the entry $a_{\mathfrak{m}}^{1}$.

All this information has been extracted from [Carlson $\geq 2004$ ]. 


\begin{tabular}{|c|c|c|c|c|c|c|c|c|c|c|c|c|c|c|c|c|c|c|c|c|c|c|c|}
\hline $\mathrm{gp}$ & $K$ & & & gp & $K$ & & & gp & & $d$ & & gp & & l & & $\mathrm{gp}$ & $K$ & $d$ & & gp & & $d$ & $r$ \\
\hline 001 & 6 & 6 & & 046 & 3 & 3 & & 091 & 4 & 3 & & 136 & 2 & 2 & & $\underline{181}$ & 3 & 1 & & 226 & 3 & 2 & \\
\hline 002 & 5 & 5 & & 047 & 4 & 3 & 3 & 092 & 3 & 3 & 3 & 137 & 2 & 2 & 1 & 182 & 2 & 1 & 1 & 227 & 2 & 2 & 2 \\
\hline 003 & 4 & 4 & 4 & 048 & 3 & 3 & 3 & 093 & 3 & 3 & 3 & 138 & 3 & 2 & 2 & $\underline{183}$ & 4 & 2 & 2 & 228 & 3 & 2 & 2 \\
\hline 004 & 4 & 4 & & 049 & 3 & 3 & 3 & $\underline{094}$ & 4 & 2 & 2 & 139 & 2 & 2 & 2 & 184 & 4 & 3 & 2 & 229 & 3 & 2 & 2 \\
\hline 005 & 3 & 3 & 3 & 050 & 3 & 3 & 3 & 095 & 3 & 2 & 2 & 140 & 2 & 2 & 2 & 185 & 3 & 2 & 2 & 230 & 3 & 2 & 2 \\
\hline 006 & 3 & 3 & & 051 & 3 & 3 & 2 & 096 & 3 & 3 & 2 & 141 & 2 & 2 & 2 & 186 & 3 & 2 & 2 & 231 & 3 & 2 & 2 \\
\hline 007 & 3 & 3 & 3 & 052 & 3 & 2 & 2 & 097 & 3 & 2 & 2 & 142 & 2 & 2 & 1 & 187 & 2 & 2 & 2 & 232 & 3 & 2 & 2 \\
\hline 008 & 2 & 2 & 2 & 053 & 3 & 3 & 2 & 098 & 3 & 2 & 2 & 143 & 2 & 1 & 1 & 188 & 3 & 3 & 2 & 233 & 2 & 2 & 2 \\
\hline 009 & 2 & 2 & 2 & 054 & 3 & 2 & 2 & 099 & 3 & 2 & 2 & 144 & 4 & 4 & 3 & 189 & 3 & 2 & 2 & 234 & 3 & 2 & 2 \\
\hline 010 & 2 & 2 & 2 & 055 & 3 & 3 & 2 & 100 & 3 & 2 & 2 & 145 & 3 & 3 & 3 & 190 & 2 & 2 & 2 & 235 & 2 & 2 & 2 \\
\hline 011 & 1 & 1 & 1 & 056 & 3 & 2 & 2 & 101 & 2 & 2 & 2 & 146 & 4 & 3 & 3 & 191 & 2 & 2 & 2 & 236 & 2 & 2 & 2 \\
\hline 012 & 5 & 5 & 4 & 057 & 2 & 2 & 2 & 102 & 3 & 2 & 2 & 147 & 4 & 3 & 3 & 192 & 2 & 2 & 2 & 237 & 3 & 3 & 2 \\
\hline 013 & 4 & 4 & 4 & 058 & 2 & 2 & 1 & 103 & 4 & 4 & 2 & 148 & 4 & 3 & 3 & 193 & 3 & 2 & 2 & 238 & 3 & 2 & 2 \\
\hline 014 & 4 & 4 & 3 & 059 & 3 & 3 & 3 & 104 & 3 & 3 & 2 & 149 & 3 & 3 & 3 & 194 & 2 & 2 & 2 & 239 & 3 & 2 & 2 \\
\hline 015 & 5 & 4 & 4 & 060 & 3 & 2 & 2 & 105 & 3 & 3 & 1 & 150 & 4 & 3 & 3 & 195 & 3 & 3 & 2 & 240 & 2 & 2 & 2 \\
\hline 016 & 4 & 4 & 4 & 061 & 3 & 2 & 2 & 106 & 4 & 3 & 2 & 151 & 4 & 3 & 3 & 196 & 3 & 2 & 2 & 44 & 3 & . & 1 \\
\hline 017 & 4 & 3 & 3 & 062 & 3 & 2 & 2 & 107 & 3 & 3 & 2 & 152 & 3 & 3 & 3 & 197 & 3 & 2 & 2 & 242 & 3 & 2 & 1 \\
\hline 018 & 4 & 4 & 3 & 063 & 2 & 2 & 2 & 108 & 3 & 2 & 2 & 153 & 3 & 3 & 3 & 198 & 3 & 2 & 2 & 243 & 2 & 2 & 1 \\
\hline 019 & 3 & 3 & 3 & 064 & 2 & 2 & 2 & 109 & 3 & 2 & 1 & 154 & 4 & 4 & 2 & 199 & 2 & 2 & 2 & 244 & 3 & 2 & 1 \\
\hline 020 & 4 & 3 & 3 & 065 & 2 & 2 & 2 & 110 & 4 & 3 & 2 & 155 & 3 & 3 & 2 & 200 & 3 & 2 & 2 & 245 & 2 & 1 & 1 \\
\hline 021 & 3 & 3 & 2 & 066 & 2 & 2 & $x$ & 111 & 3 & 2 & 2 & 156 & 2 & 2 & 2 & 201 & 4 & 5 & 2 & 246 & 2 & 1 & 1 \\
\hline 022 & 4 & 4 & 4 & 067 & 2 & 1 & 1 & 112 & 3 & 2 & 1 & 157 & 4 & 3 & 2 & $\underline{202}$ & 4 & 2 & 2 & $\underline{247}$ & 3 & 1 & 1 \\
\hline 023 & 3 & 3 & 3 & 068 & 5 & 4 & 3 & $\underline{113}$ & 4 & 2 & 2 & 158 & 3 & 3 & 2 & 203 & 3 & 3 & 2 & 248 & 2 & 2 & 1 \\
\hline 024 & 4 & 3 & 3 & 069 & 4 & 4 & 3 & $\overline{114}$ & 3 & 3 & 2 & 159 & 3 & 3 & 2 & 204 & 3 & 2 & 2 & 249 & 2 & 1 & 1 \\
\hline 025 & 3 & 3 & 3 & 070 & 3 & 3 & 3 & 115 & 3 & 2 & 2 & 160 & 3 & 3 & 2 & 205 & 3 & 3 & 2 & $\underline{250}$ & 4 & 2 & 1 \\
\hline 026 & 3 & 2 & 2 & 071 & 4 & 4 & 3 & 116 & 3 & 2 & . & 161 & 3 & 2 & 2 & 206 & 3 & 2 & 2 & $\underline{251}$ & 3 & 1 & 1 \\
\hline 027 & 3 & 3 & 2 & 072 & 4 & 3 & 3 & 117 & 3 & 3 & 2 & 162 & 2 & 2 & 2 & 207 & 3 & 2 & 2 & 252 & 3 & 2 & 1 \\
\hline 028 & 4 & 3 & 3 & 073 & 4 & 3 & 3 & 118 & 3 & 2 & 2 & 163 & 4 & 2 & 2 & 208 & 3 & 2 & 2 & 253 & 3 & 1 & 1 \\
\hline 029 & 3 & 3 & 3 & 074 & 4 & 3 & 3 & 119 & 2 & 2 & 2 & 164 & 3 & 3 & 2 & 209 & 3 & 2 & 2 & $\underline{254}$ & 3 & 1 & 1 \\
\hline 030 & 3 & 3 & 3 & 075 & 3 & 3 & 3 & 120 & 3 & 2 & 1 & 165 & 3 & 2 & 2 & 210 & 2 & 2 & 2 & 255 & 2 & 1 & 1 \\
\hline 031 & 3 & 2 & 2 & 076 & 4 & 3 & 3 & $\underline{121}$ & 3 & 1 & . & 166 & 3 & 2 & : & 211 & 2 & 2 & 2 & 256 & 3 & 2 & 1 \\
\hline 032 & 3 & 2 & 2 & 077 & 4 & 3 & 2 & 122 & 2 & 2 & 1 & 167 & 3 & 2 & 2 & 212 & 2 & 2 & 2 & $\underline{257}$ & 3 & 1 & 1 \\
\hline 033 & 3 & 2 & 2 & 078 & 3 & 3 & 2 & 123 & 4 & 3 & 2 & 168 & 3 & 2 & 2 & 213 & 3 & 2 & 2 & 258 & 2 & 1 & 1 \\
\hline 034 & 3 & 3 & 2 & 079 & 3 & 2 & 2 & $\underline{124}$ & 4 & 2 & 2 & 169 & 4 & 3 & 2 & 214 & 3 & 2 & 2 & 259 & 4 & 3 & 1 \\
\hline 035 & 2 & 2 & 2 & 080 & 3 & 2 & 2 & 125 & 3 & 2 & 2 & 170 & 4 & 3 & 2 & 215 & 3 & 2 & 2 & 260 & 3 & 2 & 1 \\
\hline 036 & 2 & 2 & 1 & $\underline{081}$ & 5 & 3 & 3 & 126 & 3 & 2 & 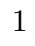 & 171 & 3 & 3 & 2 & 216 & 3 & 2 & 2 & 261 & 3 & 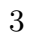 & 1 \\
\hline 037 & 3 & 3 & 3 & 082 & 3 & 3 & 3 & 127 & 3 & 2 & 1 & 172 & 3 & 2 & 2 & 217 & 3 & 3 & 2 & $\underline{262}$ & 3 & 1 & 1 \\
\hline 038 & 2 & 2 & 2 & 083 & 4 & 3 & 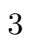 & 128 & 4 & 3 & 2 & 173 & 4 & 2 & 2 & 218 & 3 & 2 & 2 & $\overline{263}$ & 3 & 2 & 1 \\
\hline 039 & 2 & 2 & 2 & 084 & 4 & 4 & 3 & 129 & 3 & 2 & 2 & $\overline{174}$ & 3 & 2 & 2 & 219 & 3 & 2 & 2 & 264 & 2 & 2 & 1 \\
\hline 040 & 3 & 2 & 2 & 085 & 4 & 3 & 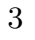 & $\underline{130}$ & 3 & 1 & 1 & $\underline{175}$ & 4 & 2 & 2 & 220 & 3 & 2 & 2 & 265 & 2 & 2 & 1 \\
\hline 041 & 2 & 2 & 2 & 086 & 4 & 3 & 3 & $\underline{131}$ & 4 & 2 & 2 & 176 & 3 & 3 & 2 & 221 & 3 & 2 & 2 & 266 & 2 & 1 & 1 \\
\hline 042 & 2 & 1 & & 087 & 3 & 3 & 8 & $\overline{132}$ & 3 & 2 & 2 & 177 & 3 & 2 & 2 & 222 & 2 & 2 & 2 & 267 & & & 1 \\
\hline 043 & 4 & 4 & 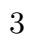 & 088 & 3 & 3 & 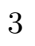 & $\underline{133}$ & 3 & 1 & 1 & 178 & 3 & 2 & 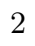 & 223 & 3 & 2 & 2 & & & & \\
\hline 044 & 4 & 3 & & 089 & 4 & 3 & & 134 & 3 & 3 & 2 & 179 & 3 & 2 & - & 224 & 3 & 2 & & & & & \\
\hline 045 & 3 & 3 & & 090 & 3 & 3 & 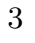 & 135 & 3 & 2 & 2 & $\underline{180}$ & 3 & 1 & 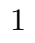 & 225 & 3 & 2 & 2 & & & & \\
\hline
\end{tabular}

Table 1. For each 2-group $G$ of order 64 , identified by its number in the notation of [Hall and Senior 1964], we give the Krull dimension $K$ of $H^{*}\left(G, \mathbb{F}_{2}\right)$, the depth $d$ of $H^{*}\left(G, \mathbb{F}_{2}\right)$, and the rank $r$ of the center of $G$. Underlined entries have $K-d=2$. Data taken from [Carlson $\geq 2004$ ]. 


\begin{tabular}{|cccccc|}
\hline Group & $a_{\mathfrak{m}}^{1}$ & $a_{\mathfrak{m}}^{2}$ & $a_{\mathfrak{m}}^{3}$ & $a_{\mathfrak{m}}^{4}$ & $a_{\mathfrak{m}}^{5}$ \\
\hline 081 & $-\infty$ & $-\infty$ & -5 & -5 & -5 \\
094 & $-\infty$ & -4 & -4 & -4 & \\
113 & $-\infty$ & -4 & -4 & -4 & \\
124 & $-\infty$ & -4 & -4 & -4 & \\
131 & $-\infty$ & -5 & -4 & -4 & \\
163 & $-\infty$ & -5 & -4 & -4 & \\
173 & $-\infty$ & -5 & -4 & -4 & \\
175 & $-\infty$ & -5 & -4 & -4 & \\
183 & $-\infty$ & -3 & -5 & -4 & \\
202 & $-\infty$ & -4 & -4 & -4 & \\
250 & $-\infty$ & -5 & -4 & -4 & \\
\hline
\end{tabular}

\begin{tabular}{|cccc|}
\hline Group & $a_{\mathfrak{m}}^{1}$ & $a_{\mathfrak{m}}^{2}$ & $a_{\mathfrak{m}}^{3}$ \\
\hline 121 & -5 & -3 & -3 \\
130 & -5 & -3 & -3 \\
133 & -5 & -3 & -3 \\
180 & -5 & -3 & -3 \\
181 & -5 & -3 & -3 \\
247 & -3 & -3 & -3 \\
251 & -4 & -3 & -3 \\
253 & -5 & -3 & -3 \\
254 & -4 & -3 & -3 \\
257 & -5 & -3 & -3 \\
262 & -5 & -3 & -3 \\
\hline
\end{tabular}

Table 2. Invariants $a_{\mathfrak{m}}^{i}\left(H^{*}\left(G, \mathbb{F}_{2}\right)\right)$ defined in (14.4), for the underlined entries of Table 1.

\section{Acknowledgements}

I thank Luchezar Avramov and Srikanth Iyengar for their many valuable comments on these notes, which have improved the exposition in a number of places. I also thank the Mathematical Sciences Research Institute for its hospitality during the Commutative Algebra Program, 2002-03.

\section{References}

[Adem and Milgram 1994] A. Adem and R. J. Milgram, Cohomology of finite groups, Grundlehren der Mathematischen Wissenschaften 309, Springer, Berlin, 1994. Second edition, 2004.

[Adem and Milgram 1995] A. Adem and R. J. Milgram, "The mod 2 cohomology rings of rank 3 simple groups are Cohen-Macaulay", pp. 3-12 in Prospects in topology (Princeton, NJ, 1994), edited by F. Quinn, Ann. of Math. Stud. 138, Princeton Univ. Press, Princeton, NJ, 1995.

[Becker and Gottlieb 1975] J. C. Becker and D. H. Gottlieb, "The transfer map and fiber bundles", Topology 14 (1975), 1-12.

[Benson 1991a] D. J. Benson, Representations and cohomology, I: Basic representation theory of finite groups and associative algebras, Cambridge Studies in Advanced Mathematics 30, Cambridge University Press, Cambridge, 1991. Paperback reprint, 1998.

[Benson 1991b] D. J. Benson, Representations and cohomology, II: Cohomology of groups and modules, Cambridge Studies in Advanced Mathematics 31, Cambridge University Press, Cambridge, 1991. Paperback reprint, 1998. 
[Benson 1993] D. J. Benson, "The image of the transfer map", Arch. Math. (Basel) 61:1 (1993), 7-11.

[Benson 1994/95] D. J. Benson, "Cohomology of modules in the principal block of a finite group", New York J. Math. 1 (1994/95), 196-205.

[Benson 2001] D. Benson, "Modules with injective cohomology, and local duality for a finite group", New York J. Math. 7 (2001), 201-215.

[Benson 2004] D. Benson, "Dickson invariants, regularity and computation in group cohomology", Illinois J. Math. 48:1 (2004), 171-197.

[Benson and Carlson 1992] D. J. Benson and J. F. Carlson, "Products in negative cohomology", J. Pure Appl. Algebra 82:2 (1992), 107-129.

[Benson and Carlson 1994a] D. J. Benson and J. F. Carlson, "Functional equations for Poincaré series in group cohomology", Bull. London Math. Soc. 26:5 (1994), 438-448.

[Benson and Carlson 1994b] D. J. Benson and J. F. Carlson, "Projective resolutions and Poincaré duality complexes", Trans. Amer. Math. Soc. 342:2 (1994), 447-488.

[Benson and Greenlees 1997a] D. J. Benson and J. P. C. Greenlees, "Commutative algebra for cohomology rings of classifying spaces of compact Lie groups", J. Pure Appl. Algebra 122:1-2 (1997), 41-53.

[Benson and Greenlees 1997b] D. J. Benson and J. P. C. Greenlees, "Commutative algebra for cohomology rings of virtual duality groups", J. Algebra 192:2 (1997), 678-700.

[Benson and Krause 2002] D. Benson and H. Krause, "Pure injectives and the spectrum of the cohomology ring of a finite group", J. Reine Angew. Math. 542 (2002), 23-51.

[Benson and Wilkerson 1995] D. J. Benson and C. W. Wilkerson, "Finite simple groups and Dickson invariants", pp. 39-50 in Homotopy theory and its applications (Cocoyoc, Mexico, 1993), edited by R. J. M. Alejandro Adem and D. C. Ravenel, Contemp. Math. 188, Amer. Math. Soc., Providence, RI, 1995.

[Benson et al. 1995] D. J. Benson, J. F. Carlson, and J. Rickard, "Complexity and varieties for infinitely generated modules", Math. Proc. Cambridge Philos. Soc. 118:2 (1995), 223-243.

[Benson et al. 1996] D. J. Benson, J. F. Carlson, and J. Rickard, "Complexity and varieties for infinitely generated modules, II", Math. Proc. Cambridge Philos. Soc. 120:4 (1996), 597-615.

[Bestvina and Feighn 2000] M. Bestvina and M. Feighn, "The topology at infinity of $\operatorname{Out}\left(F_{n}\right) "$, Invent. Math. 140:3 (2000), 651-692.

[Bieri 1976] R. Bieri, Homological dimension of discrete groups, Queen Mary College Mathematics Notes, Mathematics Department, Queen Mary College, London, 1976.

[Bøgvad and Halperin 1986] R. Bøgvad and S. Halperin, "On a conjecture of Roos", pp. 120-127 in Algebra, algebraic topology and their interactions (Stockholm, 1983), edited by J.-E. Roos, Lecture Notes in Math. 1183, Springer, Berlin, 1986.

[Borel and Serre 1973] A. Borel and J.-P. Serre, "Corners and arithmetic groups", Comment. Math. Helv. 48 (1973), 436-491.

[Bourguiba and Zarati 1997] D. Bourguiba and S. Zarati, "Depth and the Steenrod algebra", Invent. Math. 128:3 (1997), 589-602. 
[Bousfield 1979] A. K. Bousfield, "The localization of spectra with respect to homology", Topology 18:4 (1979), 257-281.

[Bousfield and Kan 1972] A. K. Bousfield and D. M. Kan, Homotopy limits, completions and localizations, Lecture Notes in Mathematics 304, Springer, Berlin, 1972.

[Broto and Henn 1993] C. Broto and H.-W. Henn, "Some remarks on central elementary abelian $p$-subgroups and cohomology of classifying spaces", Quart. J. Math. Oxford Ser. (2) 44:174 (1993), 155-163.

[Brown 1965] E. H. Brown, Jr., "Abstract homotopy theory", Trans. Amer. Math. Soc. 119 (1965), 79-85.

[Brown 1982] K. S. Brown, Cohomology of groups, Graduate Texts in Mathematics 87, Springer, New York, 1982. Corrected reprint, 1994.

[Bruner and Greenlees 2003] R. R. Bruner and J. P. C. Greenlees, The connective Ktheory of finite groups, Mem. Amer. Math. Soc. 785, Amer. Math. Soc., Providence, RI, 2003.

[Bruns and Herzog 1993] W. Bruns and J. Herzog, Cohen-Macaulay rings, Cambridge Studies in Advanced Mathematics 39, Cambridge University Press, Cambridge, 1993.

[Carlson 1981a] J. F. Carlson, "Complexity and Krull dimension", pp. 62-67 in Representations of algebras (Puebla, 1980), edited by M. Auslander and E. Lluis, Lecture Notes in Math. 903, Springer, Berlin, 1981.

[Carlson 1981b] J. F. Carlson, "The complexity and varieties of modules", pp. 415-422 in Integral representations and applications (Oberwolfach, 1980), edited by K. W. Roggenkamp, Lecture Notes in Math. 882, Springer, Berlin, 1981.

[Carlson 1983] J. F. Carlson, "The varieties and the cohomology ring of a module", J. Algebra 85:1 (1983), 104-143.

[Carlson 1987] J. F. Carlson, "Varieties and transfers", J. Pure Appl. Algebra 44:1-3 (1987), 99-105.

[Carlson 1995] J. F. Carlson, "Depth and transfer maps in the cohomology of groups", Math. Z. 218:3 (1995), 461-468.

[Carlson 1999] J. F. Carlson, "Problems in the calculation of group cohomology", pp. 107-120 in Computational methods for representations of groups and algebras (Essen, 1997), edited by P. Dräxler et al., Progr. Math. 173, Birkhäuser, Basel, 1999.

[Carlson 2001] J. F. Carlson, "Calculating group cohomology: tests for completion", J. Symbolic Comput. 31:1-2 (2001), 229-242.

[Carlson $\geq 2004]$ J. F. Carlson, "Cohomology of 2-groups of order $\leq 64$ ". Available at http://www.math.uga.edu/ jfc/groups/cohomology.html. These tables will be published in Cohomology rings of finite groups, by J. F. Carlson and L. Townsley.

[Carlson et al. 1998] J. F. Carlson, C. Peng, and W. W. Wheeler, "Transfer maps and virtual projectivity", J. Algebra 204:1 (1998), 286-311.

[Cartan and Eilenberg 1956] H. Cartan and S. Eilenberg, Homological algebra, Princeton University Press, Princeton, NJ, 1956.

[Dickson 1911] L. E. Dickson, "A fundamental system of invariants of the general modular linear group with a solution of the form problem", Trans. Amer. Math. Soc. 12 (1911), 75-98. 
[Duflot 1981] J. Duflot, "Depth and equivariant cohomology", Comment. Math. Helv. 56:4 (1981), 627-637.

[Dwyer and Wilkerson 1994] W. G. Dwyer and C. W. Wilkerson, "Homotopy fixedpoint methods for Lie groups and finite loop spaces", Ann. of Math. (2) 139:2 (1994), 395-442.

[Dwyer et al. 2002] W. G. Dwyer, J. P. C. Greenlees, and S. Iyengar, "Duality in algebra and topology", Preprint, University of Notre Dame, 2002. Available at http://www.nd.edu/ wgd.

[Eisenbud 1995] D. Eisenbud, Commutative algebra, with a view toward algebraic geometry, Graduate Texts in Mathematics 150, Springer, New York, 1995.

[Eisenbud and Goto 1984] D. Eisenbud and S. Goto, "Linear free resolutions and minimal multiplicity", J. Algebra 88:1 (1984), 89-133.

[Elmendorf et al. 1997] A. D. Elmendorf, I. Kříž, M. A. Mandell, and J. P. May, Rings, modules, and algebras in stable homotopy theory, Mathematical Surveys and Monographs, American Mathematical Society, Providence, RI, 1997.

[Evens 1961] L. Evens, "The cohomology ring of a finite group", Trans. Amer. Math. Soc. 101 (1961), 224-239.

[Evens 1991] L. Evens, The cohomology of groups, Oxford Mathematical Monographs, The Clarendon Press Oxford University Press, New York, 1991. Oxford Science Publications.

[Fiedorowicz and Priddy 1978] Z. Fiedorowicz and S. Priddy, Homology of classical groups over finite fields and their associated infinite loop spaces, Lecture Notes in Mathematics 674, Springer, Berlin, 1978.

[Friedlander and Suslin 1997] E. M. Friedlander and A. Suslin, "Cohomology of finite group schemes over a field", Invent. Math. 127:2 (1997), 209-270.

[Golod 1959] E. Golod, "The cohomology ring of a finite p-group", Dokl. Akad. Nauk SSSR 125 (1959), 703-706. In Russian.

[Green 2003] D. J. Green, "On Carlson's depth conjecture in group cohomology", Math. Z. 244:4 (2003), 711-723.

[Greenlees 1995] J. P. C. Greenlees, "Commutative algebra in group cohomology", J. Pure Appl. Algebra 98:2 (1995), 151-162.

[Greenlees 2002] J. P. C. Greenlees, "Local cohomology in equivariant topology", pp. 1-38 in Local cohomology and its applications (Guanajuato, 1999), edited by G. Lyubeznik, Lecture Notes in Pure and Appl. Math. 226, Dekker, New York, 2002.

[Greenlees and Lyubeznik 2000] J. P. C. Greenlees and G. Lyubeznik, "Rings with a local cohomology theorem and applications to cohomology rings of groups", J. Pure Appl. Algebra 149:3 (2000), 267-285.

[Grothendieck 1965] A. Grothendieck, Cohomologie locale des faisceaux cohérents et théorèmes de Lefschetz locaux et globaux, Séminaire de géométrie algébrique $\mathbf{2}$, Institut des Hautes Études Scientifiques, Paris, 1965. Reprinted North-Holland, Amsterdam, 1968, Advanced Studies in Pure Math 2.

[Grothendieck 1967] A. Grothendieck, Local cohomology, Lecture Notes in Math. 41, Springer, Berlin, 1967. 
[Hall and Senior 1964] M. Hall, Jr. and J. K. Senior, The groups of order $2^{n}(n \leq 6)$, The Macmillan Co., New York, 1964.

[Harer 1986] J. L. Harer, "The virtual cohomological dimension of the mapping class group of an orientable surface", Invent. Math. 84:1 (1986), 157-176.

[Iyengar 2004] S. Iyengar, "Modules and cohomology over group algebras: One commutative algebraist's perspective", pp. 51-86 in Trends in Commutative Algebra, edited by L. Avramov et al., Math. Sci. Res. Inst. Publ. 51, Cambridge University Press, New York, 2004.

[Kleinerman 1982] S. N. Kleinerman, The cohomology of Chevalley groups of exceptional Lie type, Mem. Amer. Math. Soc. 268, Amer. Math. Soc., Providence, RI, 1982.

[Kono and Mimura 1975] A. Kono and M. Mimura, "Cohomology $\bmod 2$ of the classifying space of the compact connected Lie group of type $E_{6}$ ", J. Pure Appl. Algebra 6 (1975), 61-81.

[Lazard 1965] M. Lazard, "Groupes analytiques p-adiques", Inst. Hautes Études Sci. Publ. Math. 26 (1965), 389-603.

[Matlis 1958] E. Matlis, "Injective modules over Noetherian rings", Pacific J. Math. 8 (1958), 511-528.

[Matsumura 1989] H. Matsumura, Commutative ring theory, Second ed., Cambridge Studies in Advanced Mathematics 8, Cambridge University Press, Cambridge, 1989. Translated from the Japanese by M. Reid.

[Milnor 1956] J. Milnor, "Construction of universal bundles, II", Ann. of Math. (2) 63 (1956), 430-436.

[Minh and Symonds 2004] P. A. Minh and P. Symonds, "Cohomology and finite subgroups of profinite groups", Proc. Amer. Math. Soc. 132 (2004), 1581-1588.

[Nagata 1962] M. Nagata, Local rings, Interscience Tracts in Pure and Applied Mathematics, No. 13, Interscience Publishers a division of John Wiley \& Sons New YorkLondon, 1962. Corrected reprint, Robert E. Krieger Publishing Co., Huntington, N.Y., 1975.

[Neeman 1996] A. Neeman, "The Grothendieck duality theorem via Bousfield's techniques and Brown representability", J. Amer. Math. Soc. 9:1 (1996), 205-236.

[Neusel 2000] M. D. Neusel, Inverse invariant theory and Steenrod operations, Mem. Amer. Math. Soc. 692, Amer. Math. Soc., Providence, RI, 2000.

[Okuyama and Sasaki 2000] T. Okuyama and H. Sasaki, 2000. Private communication, Chiba, Japan.

[Quillen 1971a] D. Quillen, "The mod 2 cohomology rings of extra-special 2-groups and the spinor groups", Math. Ann. 194 (1971), 197-212.

[Quillen 1971b] D. Quillen, "The spectrum of an equivariant cohomology ring, I", Ann. of Math. (2) 94 (1971), 549-572.

[Quillen 1971c] D. Quillen, "The spectrum of an equivariant cohomology ring, II", Ann. of Math. (2) 94 (1971), 573-602.

[Quillen 1972] D. Quillen, "On the cohomology and $K$-theory of the general linear groups over a finite field", Ann. of Math. (2) 96 (1972), 552-586. 
[Rickard 1997] J. Rickard, "Idempotent modules in the stable category", J. London Math. Soc. (2) 56:1 (1997), 149-170.

[Rusin 1989] D. J. Rusin, "The cohomology of the groups of order 32", Math. Comp. 53:187 (1989), 359-385.

[Schwartz 1994] L. Schwartz, Unstable modules over the Steenrod algebra and Sullivan's fixed point set conjecture, Chicago Lectures in Mathematics, University of Chicago Press, Chicago, IL, 1994.

[Serre 1965a] J.-P. Serre, Cohomologie galoisienne, 3rd ed., Lecture Notes in Mathematics 5, Springer, Berlin, 1965.

[Serre 1965b] J.-P. Serre, "Sur la dimension cohomologique des groupes profinis", Topology 3 (1965), 413-420.

[Sri] Srikanth Iyengar's article in this volume; details under [Iyengar 2004].

[Steenrod 1962] N. E. Steenrod, Cohomology operations, Annals of Mathematics Studies 50, Princeton University Press, Princeton, 1962. Notes written and revised by D. B. A. Epstein.

[Venkov 1959] B. B. Venkov, "Cohomology algebras for some classifying spaces", Dokl. Akad. Nauk SSSR 127 (1959), 943-944. in Russian.

[Wilkerson 1982] C. Wilkerson, Spring 1982. Letter to Frank Adams.

[Wilkerson 1983] C. Wilkerson, "A primer on the Dickson invariants", pp. 421-434 in Proceedings of the Northwestern Homotopy Theory Conference (Evanston, IL, 1982), edited by H. R. Miller and S. B. Priddy, Contemp. Math. 19, Amer. Math. Soc., Providence, RI, 1983.

[Ziegler 1984] M. Ziegler, "Model theory of modules", Ann. Pure Appl. Logic 26:2 (1984), 149-213.

Dave Benson

Department of MATHEMATICS

University of GeORGiA

ATHENS GA 30602

UNited STATES

bensondj@math.uga.edu 